\title{
Superconducting Materials for Large Scale Applications
}

\author{
RONALD M. SCANLAN, ALEXIS P. MALOZEMOFF, SENIOR MEMBER, IEEE, AND \\ DAVID C. LARBALESTIER
}

\section{Invited Paper}

Since the 1960s, Nb-Ti (superconducting transition temperature $\left.T_{c}=9 \mathrm{~K}\right)$ and $\mathrm{Nb}_{3} \mathrm{Sn}\left(T_{c}=18 \mathrm{~K}\right)$ have been the materials of choice for virtually all superconducting magnets. However, the prospects for the future changed dramatically in 1987 with the discovery of layered cuprate superconductors with $T_{c}$ values that now extend up to about $135 \mathrm{~K}$. Fabrication of useful conductors out of the cuprates has been difficult, but a first generation of silver-sheathed composite conductors based on $(\mathrm{Bi}, \mathrm{Pb})_{2} \mathrm{Sr}_{2} \mathrm{Ca}_{2} \mathrm{Cu}_{3} \mathrm{O}_{10}\left(\mathrm{~T}_{c} \sim 110 \mathrm{~K}\right)$ has already been commercialized. Recent progress on a second generation of biaxially aligned coated conductors using the less anisotropic $\mathrm{YBa}_{2} \mathrm{Cu}_{3} \mathrm{O}_{7}$ structure has been rapid, suggesting that it too might enter service in the near future. The discovery of superconductivity in $\mathrm{MgB}_{2}$ below $39 \mathrm{~K}$ in 2001 has brought yet another candidate material to the large-scale applications mix. Two distinct markets for superconductor wires exist-the more classical low-temperature magnet applications such as particle accelerators, nuclear magnetic resonance and magnetic resonance imaging magnets, and plasma-containment magnets for fusion power, and the newer and potentially much larger market for electric power equipment, such as motors, generators, synchronous condensers, power transmission cables, transformers, and fault-current limiters for the electric utility grid. We review key properties and recent progress in these materials and assess their prospects for further development and application.

Keywords- $\mathrm{Bi}_{2} \mathrm{Sr}_{2} \mathrm{CaCu}_{2} \mathrm{O}_{8}$, $(\mathrm{Bi}, \mathrm{Pb})_{2} \mathrm{Sr}_{2} \mathrm{Ca}_{2} \mathrm{Cu}_{3} \mathrm{O}_{10}$, BSCCO-2212, BSCCO-2223, coated conductors, $\mathrm{MgB}_{2}, \mathrm{Nb}_{3} \mathrm{Sn}$, $\mathrm{Nb}-\mathrm{Ti}$, superconducting wires, superconductors, $\mathrm{YBa}_{2} \mathrm{Cu}_{3} \mathrm{O}_{7-\delta}$, $\mathrm{YBa}_{2} \mathrm{Cu}_{3} \mathrm{O}_{7}(\mathrm{YBCO})$.

Manuscript received December 15, 2003; revised May 4, 2004.

R. M. Scanlan, retired, was with the Lawrence Berkeley National Laboratory, Berkeley, CA 94720 USA. He is now at 26a Lost Valley Drive, Orinda, CA 94563 USA (e-mail: rmscanlan@aol.com).

A. P. Malozemoff is with the American Superconductor Corporation, Westborough, MA 01581 USA (e-mail: amalozemoff@amsuper.com).

D. C. Larbalestier is with the Applied Superconductivity Center, University of Wisconsin, Madison, WI 53706-1609 USA (e-mail: larbalestier@engr.wisc.edu).

Digital Object Identifier 10.1109/JPROC.2004.833673

\section{INTRODUCTION}

Superconducting conductors for electric power and magnet applications are poised for major change. For about 40 years, virtually all magnets have been made out of $\mathrm{Nb}-\mathrm{Ti}$ or $\mathrm{Nb}_{3} \mathrm{Sn}$, the cubic low-temperature superconductors (LTSs), which possess transition temperatures $T_{c}$ of $9 \mathrm{~K}$ and $18 \mathrm{~K}$, respectively [1]. In spite of the much higher $T_{c}$ values of high-temperature superconductor (HTS) materials, $\sim 90 \mathrm{~K}$ (BSCCO-2212 and YBCO-123) and $\sim 110 \mathrm{~K}$ (BSCCO-2223), and strong progress in developing HTS wire, these materials have not yet made a serious dent in the commercial dominance of $\mathrm{Nb}-\mathrm{Ti}$ and $\mathrm{Nb}_{3} \mathrm{Sn}$ in the broader magnet market [1]. That market has for years consisted of magnetic resonance imaging (MRI) and nuclear magnetic resonance (NMR), and magnets for high-energy physics accelerators and plasma fusion devices, together with smaller niches for research magnets ${ }^{1}$ [2]. These applications were developed with LTS conductors and are well served by their low cost, coupled with their ability to be fabricated as strong, round wires with distinct filament structures, high current density and a high superconductor fill factor.

$\mathrm{Nb}-\mathrm{Ti}$ conductors were scientifically understood and commercially optimized during the $1980 \mathrm{~s}$. Nb-Ti is in fact the only material whose fabrication process has been effectively optimized based on a detailed scientific understanding. This understanding is still being developed for the high-field use of $\mathrm{Nb}_{3} \mathrm{Sn}$, especially for NMR and particle accelerator applications, with much recent progress.

Since the late 1980s, conductors made from the silver-sheathed $\mathrm{HTSs} \quad \mathrm{Bi}_{2} \mathrm{Sr}_{2} \mathrm{CaCu}_{2} \mathrm{O}_{8}$ (BSCCO-2212) and $(\mathrm{Bi}, \mathrm{Pb})_{2} \mathrm{Sr}_{2} \mathrm{Ca}_{2} \mathrm{Cu}_{3} \mathrm{O}_{10}$ (BSCCO-2223) have been in ongoing development [1]. Although the understanding of their materials science is still limited and process optimization is for now rather empirical, they have progressed to the point of being commercially available, with acceptable

${ }^{1}$ See also other papers in this special issue. 
mechanical properties and practical current densities in the 30-77 $\mathrm{K}$ range [3]. These are known as first-generation HTS wires or conductors, and they are principally directed at electric power applications, a major new market for superconductivity ${ }^{2}$ [4]. Conductors made of BSCCO-2223 are being applied in significant prototypes of electric power equipment, including an 8-MVA reactive (MVAR) synchronous condenser [5], a 5000-hp industrial motor [5], a 5 MW high-torque ship propulsion motor [5], a 5-MVA transformer [6], and a variety of power transmission and distribution cables [7]. Very recently, a magnet of BSCCO-2212 wire has generated $5 \mathrm{~T}$ in a background field of $20 \mathrm{~T}$, making it the highest field superconducting magnet [8], a result which clearly shows the potential of HTS materials to overtake LTS materials in magnet applications.

Simultaneously, the HTS material $\mathrm{RBa}_{2} \mathrm{Cu}_{3} \mathrm{O}_{7}$, where $\mathrm{R}$ is a rare-earth atom or yttrium, has been developed in a coated conductor format [9], [10]; we will use $\mathrm{YBCO}$ to denote this material family, since $\mathrm{YBa}_{2} \mathrm{Cu}_{3} \mathrm{O}_{7}$ is the most commonly used member. This technology is rapidly moving from research and development to scale-up and has already enabled a first short power cable prototype carrying a commercial-level current [11]. Coated conductors are often called second-generation HTS wires or conductors. In addition, some developmental conductors are starting to be fabricated from the recently discovered superconducting material $\mathrm{MgB}_{2}$ [12].

The situation in 2004 is distinctly different than that of five years ago. For the first time, one can say that the Nb-based LTS conductors are approaching their limits, and that BSCCO and YBCO materials, and perhaps $\mathrm{MgB}_{2}$, are ready to surpass LTS capabilities in higher field and higher temperature domains, thus opening up new markets.

The largest potential market for HTS conductors lies in the electric power arena and involves power transmission cables, high-power industrial and ship propulsion motors, utility generators, synchronous condensers, fault-current limiters, and transformers. Study after study, such as Vice President Cheney's Task Force on National Energy Policy [13], the Secretary of Energy's National Transmission Grid Study [14], and Grid 2030 [15], the vision of the future transmission system from the Department of Energy (DOE) Office of Electric Transmission and Distribution, have highlighted the critical reliability and capacity issues in the U.S. power grid and specifically noted the beneficial potential impact of superconducting technology in addressing these issues. The Northeast blackout of Aug. 14, 2003, has elevated calls for action. Momentum from these events is propelling HTS conductors into commercialization.

In this paper, we review key properties and technical progress in superconducting materials for large-scale applications during the last few years and assess their prospects for further development and application. We limit discussion to materials in the form of flexible, long-length wires rather than bulk pellets and rods, which have their own array of issues and applications.

${ }^{2}$ See also other papers in this special issue.

\section{CONDUCTOR REQUiREMENTS FOR POWER AND MAGNET TECHNOLOGY}

Superconducting conductors for large-scale applications are round or tape-shaped wires in which one or more superconducting filaments are embedded in a matrix consisting at least partially of a normal metal, such as $\mathrm{Cu}$ or $\mathrm{Ag}$, which provides protection against magnetic flux jumps and thermal quenching [1], [16] (Fig. 1). Such wires must have sufficient strength to withstand conductor fabrication, coil winding, cabling, the thermal stresses of cooldown, and operational electromagnetic stresses. They must be capable of carrying operating currents, dc or ac, of hundreds of amperes and, often, of being cabled to carry thousands of amperes. In particular, the minimum critical tensile stress before loss of critical current density should be in the range of $100 \mathrm{MPa}$ or higher, and minimum tensile, compressive, and bending strains before degradation must be several tenths of a percent. Engineering (total cross section) current density $J_{e}$ must attain $10^{4}-10^{5} \mathrm{~A} / \mathrm{cm}^{2}$ in magnetic fields which extend from $\sim 0.1$ to $25 \mathrm{~T}$, depending on the application. The superconductor current density $J_{c}$ is $J_{e} / f$, where $f$ is the fill fraction of superconducting material in the total conductor cross section. Operating temperatures tend to be as low as possible for ultrahigh-field ( $\sim 25 \mathrm{~T}) \mathrm{dc}$ applications (for example, $2 \mathrm{~K}$ in superfluid helium) but are as high as possible for ac power applications, since ac losses generated at low temperatures exact a stiff cooling penalty of many times the Carnot efficiency in practical cryogenic environments.

Estimates of the maximum acceptable price for different applications range from $\sim \$ 1$ to 100 per kiloamp-meter, where kiloamp (kA) refers to the operating current level. In particular, $\mathrm{Nb}-\mathrm{Ti}$ wire typically sells for $\$ 1 / \mathrm{kAm}$ but is limited to operation in the helium temperature range. HTSs operating at $20-77 \mathrm{~K}$ are expected to be economical for some applications in the $\$ 10-100 / \mathrm{kAm}$ range. In power equipment, copper wires typically operate in the range of $\$ 15-25 / \mathrm{kAm}$; this sets a benchmark for superconducting wire. As a whole, LTS conductors are used for applications where few or no conventional alternatives exist, while HTS materials must compete against copper in electric power technology, where cost pressures are almost always significant.

\section{BASIC Physical Properties OF SuPERCONDUCTING WIRES}

We give only a brief summary of the basic physical concepts underlying the performance of superconducting materials in conductors; these are described more extensively elsewhere [1], [9], [16], [17]. These materials exhibit superconducting properties in a region below the interdependent values of critical temperature $T_{c}$, upper critical magnetic field $H_{c 2}$, and critical current density $J_{c}$. The maximum critical temperature for metallic LTSs is $23 \mathrm{~K}$; it is $40 \mathrm{~K}$ for $\mathrm{MgB}_{2}$ [12] and about $135 \mathrm{~K}$ for HTSs based on cuprate (copper oxide) compositions [18].

Fig. 2 presents the magnetic-field/temperature $(H-T)$ phase diagram for the three currently commercial conductor 


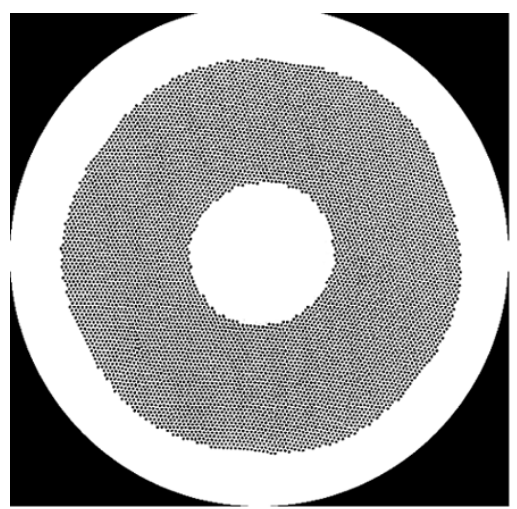

(a)

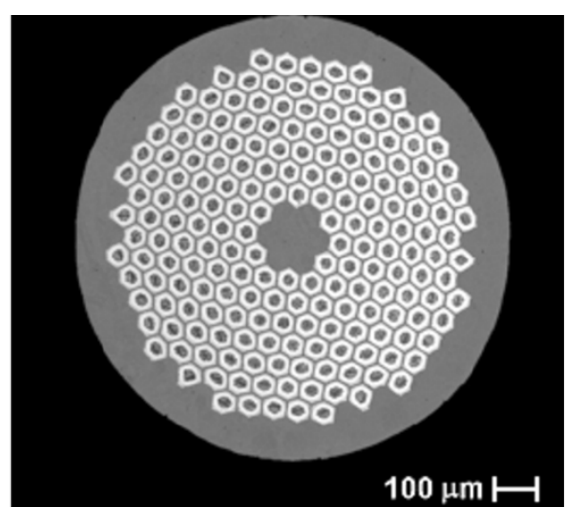

(b)

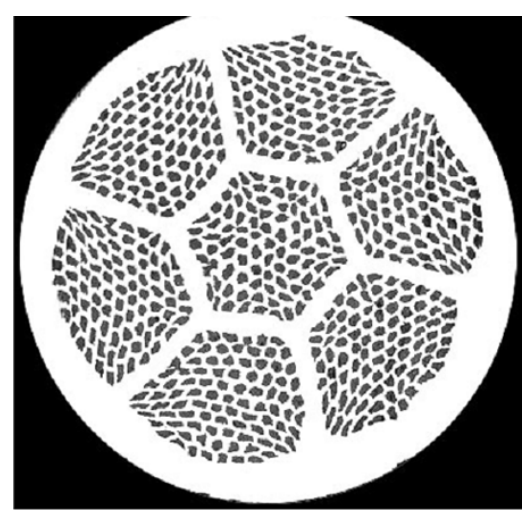

(c)

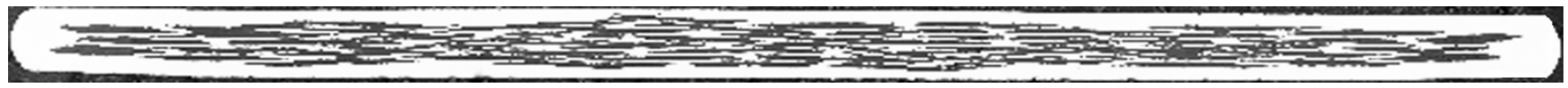

(d)

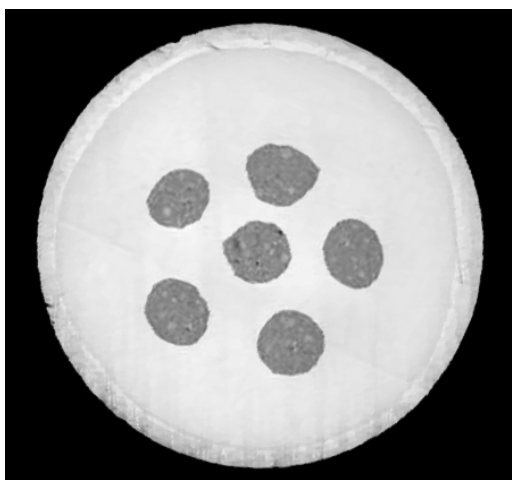

(e)

Fig. 1. Representative multifilamentary conductors made from: (a) Nb47wt.\% Ti; (b) $\mathrm{Nb}_{3} \mathrm{Sn}$; (c) $\mathrm{Bi}-2212$; (d) $\mathrm{Bi}-2223$; and (e) $\mathrm{MgB}_{2}$. The matrices for the conductors are high-purity copper for (a) and (b) and the outer sheath of (e) and pure silver or silver alloy for (c) and (d). The filaments of $\mathrm{MgB}_{2}$ are surrounded by 316 stainless steel in (e). Conductors were manufactured by: (a) and (c) Oxford Instruments-Superconducting Technology; (b) ShapeMetal Innovation; (d) American Superconductor Corporation; and (e) Hitachi Cable in collaboration with the National Institute for Materials Science.

materials: $\mathrm{Nb}-\mathrm{Ti}, \quad \mathrm{Nb}_{3} \mathrm{Sn}$, and $(\mathrm{Bi}, \mathrm{Pb})_{2} \mathrm{Sr}_{2} \mathrm{Ca}_{2} \mathrm{Cu}_{3} \mathrm{O}_{10}$ (BSCCO-2223), and developing conductor materials: $\mathrm{YBa}_{2} \mathrm{Cu}_{3} \mathrm{O}_{7}$ (YBCO) and $\mathrm{MgB}_{2}$. Their very different phase diagrams result from their distinctly different crystal structures and physical parameters. All six are type II superconductors for which bulk superconductivity exists up to an upper critical field $H_{c 2}(T)$, that can be highly anisotropic and exceeds $100 \mathrm{~T}$ for BSCCO-2223 and YBCO. However, applications of HTS materials are limited by a lower characteristic field, the so-called "irreversibility field" $H^{*}(T)$, at which the bulk $J_{c}$ vanishes [19].

Under equilibrium conditions magnetic flux penetrates the bulk of a Type II superconductor above a lower critical field $H_{c 1}$, which is $<100 \mathrm{mT}$ for the materials under consideration [19]. This magnetic flux exists as an array of flux-quantized line vortices or fluxons, which can be spatially ordered or disordered, static or liquid-like. Each vortex is a tube of radius of the London penetration depth $\lambda(T)$, in which superconducting screening currents circulate around a small nonsuperconducting core of radius $\xi(T)$, where $\xi(T)$ is the superconducting coherence length. The flux carried by the screening currents in each vortex equals the flux quantum $\phi_{0}=2 \times 10^{-15} \mathrm{~Wb}$. Bulk superconductivity disappears when the normal cores overlap at the upper critical field $H_{c 2}(T)=\phi_{0} / 2 \pi \mu_{0} \xi(T)^{2}$.

Type II superconductors can carry bulk current density only if there is a macroscopic fluxon density gradient defined by the Maxwell equation $\nabla \times \mathbf{B}=\mu_{0} \mathbf{J}$. This gradient must be sustained by the pinning of vortices at microstructural defects, and much process development is oriented to optimizing this "flux pinning" to maximize the current carrying capacity. At absolute zero, superconducting current can flow without any loss (i.e., at zero voltage) up to a critical current $J_{c}$, corresponding to the maximum pinning strength. However, at finite temperature, thermal activation can cause "flux creep" of vortices down the macroscopic fluxon density gradient (actually a small amount of creep can occur even at absolute zero because of quantum tunneling of vortices, observed in HTS materials) [19]-[21]. In most materials with random microstructural defects, the vortex structure is disordered or glassy in nature, and vortex glass theory predicts that flux creep gives rise to a $V \sim I^{n}$ 


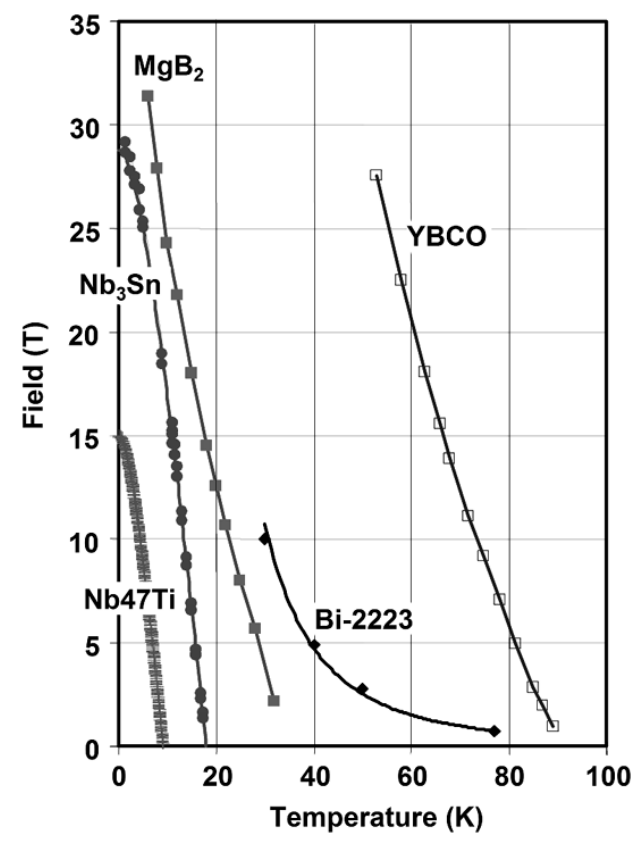

Fig. 2. Upper critical field $\left(H_{c 2}\right)$ for $\mathrm{Nb} 47 \mathrm{wt} . \% \mathrm{Ti}, \mathrm{Nb}_{3} \mathrm{Sn}$, and $\mathrm{MgB}_{2}$, and irreversibility fields $\left(H^{*}\right)$ for Bi-2223, and $\mathrm{YBCO}$. Note that the two fields are very close for $\mathrm{Nb} 47 \mathrm{wt} . \% \mathrm{Ti}, \mathrm{Nb}_{3} \mathrm{Sn}$, and $\mathrm{MgB}_{2},\left(H^{*}\right.$ is $85 \%-90 \%$ of $\left.H_{c 2}\right)$ but widely separated for all cuprate superconductors. For $\mathrm{MgB}_{2}, \mathrm{Bi}-2223$, and $\mathrm{YBCO}$, the values plotted are the lower values appropriate to fields perpendicular to the strongest superconducting planes, the $\mathrm{B}$ planes for $\mathrm{MgB}_{2}$ and the $\mathrm{CuO}_{2}$ planes in the cuprates. Values plotted are the highest credible for each compound.

voltage-current relationship, where $n$ is called the "index value" [20], [21]. Nonuniformities in material properties on nanometer or greater length scales can also give rise to this power-law relationship. The index value must be well above 30 to allow the superconducting wire to operate in a quasi-persistent mode, with minimal current decay over time. This condition can be achieved in $\mathrm{Nb}-\mathrm{Ti}$ and $\mathrm{Nb}_{3} \mathrm{Sn}$ but only rarely in HTS wires where typical index values range from 15 to 30 . Because the voltage-current curve is continuous, there is no absolute measure of $J_{c}$. However, voltage increases rapidly enough with current to enable a practical definition of $J_{c}$ at a characteristic electric field, typically chosen to be either 0.1 or $1 \mu \mathrm{V} / \mathrm{cm}$. For magnet applications, even lower electric fields may be appropriate.

Above $J_{c}$, or at magnetic fields above a characteristic "irreversibility field" $H^{*}$, which is a function of temperature and which is much lower than $H_{c 2}$ for HTS materials, major changes occur in the vortex structure [19]. These changes have been described by a variety of theories-for example, the vortex glass melting theory, in which vortices above $H^{*}$ are in an unpinned, liquid-like state. Under these conditions, current will drive a dissipative, flux-flow state, with a resistivity which, to first approximation, is given by the fraction of the material occupied by the normal cores of the vortices. This simple concept leads directly to the Bardeen-Stephen formula for flux flow resistivity $\rho_{\mathrm{FF}}=\rho_{N} B / \mu_{0} H_{c 2}$, where $\rho_{N}$ is the normal state resistivity [17].

Long conductors for bulk applications are always based on polycrystalline materials, and the influence of grain bound-

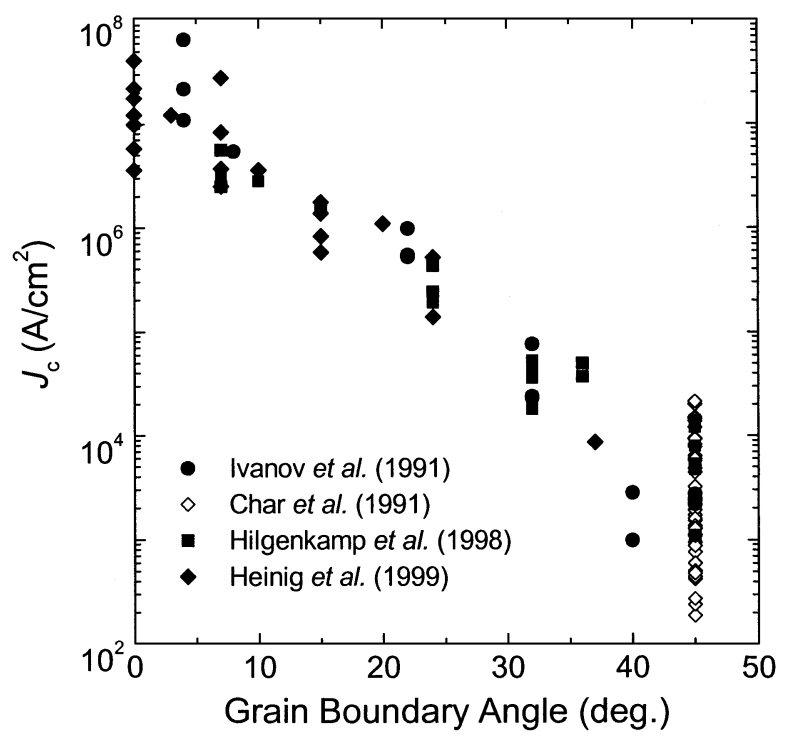

Fig. 3. Critical current density across [001] tilt grain boundaries of YBCO-123 measured at $4.2 \mathrm{~K}$ and self-field, from multiple studies on bicrystal boundaries [25]. The exponential falloff of $J_{c}$ with grain boundary angle creates the underlying requirement for texturing to achieve high currents in HTS wires. (Based on original work of Dimos et al. [24]).

aries is, thus, decisive. In metallic LTS and $\mathrm{MgB}_{2}$, current flow is uniform through the polycrystalline superconducting matrix unless gross obstructions such as cracks occur. In fact, grain boundaries are often beneficial flux pinning centers, so that $J_{c}$ typically increases as the grain size decreases [22].

However, HTS materials have low carrier density and short coherence lengths, factors that predispose grain boundaries to act as obstacles to current flow [23]. The most unambiguous evidence for this comes from detailed studies of YBCO films grown epitaxially on bicrystals of $\mathrm{SrTiO}_{3}$ or $\mathrm{Y}_{2} \mathrm{O}_{3}$ - stabilized $\mathrm{ZrO}_{2}$ (YSZ) substrates. The critical current density $J_{b}$ across the grain boundary drops exponentially below that within the grains, $J_{b}=J_{c} \exp \left(-\theta / \theta_{c}\right)$, as a function of the misorientation angle $\theta$ between the neighboring crystallites [24]-[26], where $\theta_{c} \approx 2-5^{\circ}$, depending on the value of the intragrain $J_{c}$ (Fig. 3).

At angles of about $10^{\circ}$ and below, the structure of the grain boundary breaks up into identifiable edge dislocations spaced by $b / 2 \sin (\theta / 2)$, where $b \approx 0.4 \mathrm{~nm}$ is the Burgers vector. The cores of these dislocations in HTS materials are insulating. Above a misorientation angle $\theta$ of $5-7^{\circ}$, the spacing between the cores is less than the coherence length $\xi$, and the grain boundary starts to behave like a Josephson weak link. At lower misorientation angles, the dislocation cores are separated by only modestly strained material, which enables strong coupling and current flow between the grains at the bulk $J_{c}$ level. The possibility of fabricating highly textured substrates coated with an epitaxial HTS layer, in which $\theta$ is small enough to permit strong coupling and, hence, significant supercurrent flow, is the basis for the coated conductor class of HTS wire [9].

The mechanism of current flow across grain boundaries in BSCCO-based conductors may be different [27], [28]. Although these materials are strongly textured around a 
common $c$ axis in wires, the in-plane orientations of their grains' $a b$ planes are random, causing direct lateral current flow across high-angle grain boundaries to be exponentially weak. The observed current flow appears instead to involve redistribution of the current across the large-area twist boundaries separating $c$ axis-aligned grains, allowing current to bypass high-angle grain boundaries and other obstacles.

The measurement of critical current for LTS and HTS conductors has evolved in distinctly different ways. LTS wires are always tested in magnetic fields appropriate to the application, e.g., 5-7 $\mathrm{T}$ for $\mathrm{Nb}-\mathrm{Ti}$ and $10-15 \mathrm{~T}$ for $\mathrm{Nb}_{3} \mathrm{Sn}$ at an electric field criterion that approximates magnet performance, often $0.1 \mu \mathrm{V} / \mathrm{cm}$ [29]. However the standard measurement for HTS materials, because of its simplicity, is in self-field at $77 \mathrm{~K}$ at $1 \mu \mathrm{V} / \mathrm{cm}$. The drawback of this test is that $J_{c}$ drops rapidly with even weak applied or self-magnetic fields, making results dependent on the conductor geometries. In the case of BSCCO-2223 conductors, proper comparison to avoid these self-field effects requires a field of at least $0.1 \mathrm{~T}$ at $77 \mathrm{~K}$ [30]. As applications develop, testing appropriate to the operating conditions will be established.

Under conditions of time-varying current and/or magnetic field, superconductors are subject to ac losses. There are three types of losses in addition to the usual eddy current losses of the normal metal matrix: 1) hysteretic, due to the motion of vortices through the superconductor; 2) interfilament "coupling" losses, due to induced currents flowing between filaments across the normal metal matrix within a multifilamentary strand; and 3) interstrand "coupling" losses, due to eddy currents flowing between the stands in a multistrand conductor (see [16]). These losses can be mitigated, but not completely eliminated, as follows. In low magnetic field environments in which the superconductor is only partially penetrated by the ac magnetic flux, the ac loss varies inversely with $J_{c}$ and, therefore, can be mitigated by increasing $J_{c}$. In high-field environments in which the superconductor is fully penetrated by ac magnetic flux, hysteretic loss, normalized to the current capacity of the wire, is proportional to $d$, the superconductor filament diameter (or width perpendicular to the applied field), provided the filaments are decoupled. Thus, practical superconductors for ac applications are subdivided into fine filaments imbedded in a normal metal matrix so that $d$ is reduced to a small fraction of the wire diameter (Fig. 1). Round configurations are favorable compared to tape-shaped wires, unless ac fields can be oriented along the wide face of the tapes to minimize $d$; round wires facilitate cabling with transposition and reduce the maximum transverse dimension as compared to a tape.

Typical multifilamentary superconductors may contain from several tens (MRI magnet) to several thousands of filaments (NMR, fusion, or accelerator magnets), with diameters from $\sim 50$ to $0.1 \mu \mathrm{m}$, depending upon the application. For $\mathrm{Nb}-\mathrm{Ti}$, the effective filament size $d_{\text {eff }}$ is usually the physical filament diameter. However, for $\mathrm{Nb}_{3} \mathrm{Sn}$ and all multifilamentary HTS conductors, the effective filament size can be much larger, since the filaments may coalesce during the reaction step needed to produce $\mathrm{Nb}_{3} \mathrm{Sn}$, while for BSCCO-2212 or BSCCO-2223, superconducting grains grow through the Ag matrix to form a superconducting path between adjacent filaments.

Because the matrix material is typically a good normal metal such as $\mathrm{Cu}$ in LTS or Ag in HTS, the interfilamentary resistance is typically low enough under magnet-charging rates to allow coupling currents to flow between filaments and, thus, shield the interior of the wire from flux changes. Twisting the conductor or transposing the filaments can reduce these coupling currents, so that the magnitude of the induced electromotive force driving the currents is lessened and periodically reverses along the wire length. Typical twist pitches are ten times the wire diameter, with a ratio of five being the minimum possible. For large magnets requiring high currents, the conductor must be built up by cabling many strands together, preferably in a fully transposed geometry. The interstrand resistance then controls the magnitude of strand-to-strand coupling currents. This source of loss can be reduced by increasing the interstrand resistance, for example, by adding a high resistance alloy layer such as $\mathrm{Cu}-\mathrm{Ni}$ or a Cr-plate in LTS conductors, or by applying an insulating coating to the strands.

One final issue of importance for conductor properties concerns whether current is percolative or flows uniformly through the whole cross section. For a uniform conductor, division of the measured critical current by the cross-sectional area of superconductor $A_{\mathrm{sc}}$ then gives the intrinsic critical current density $J_{c}$ determined by the flux pinning strength of the material. For $\mathrm{Nb}-\mathrm{Ti}$ and $\mathrm{Nb}_{3} \mathrm{Sn}$ certain extrinsic defects to be described later affect the value of $A_{\mathrm{sc}}$ but introduce an error in calculating $J_{c}$ of no more than $5 \%-10 \%$. For HTS materials, this error may be much larger, because of percolative current flow arising from a combination of intrinsic obstacles like grain boundaries and extrinsic defects such as large insulating second-phase particles or cracks [9]. One of the central tasks of conductor development has been to understand such obstacles to current flow and to eliminate them as best one can, finally asking how close $J_{c}$ can be brought to the ultimate possible current density, which is the depairing current density of the vortex currents $J_{d}$ [17]. In $\mathrm{Nb}-\mathrm{Ti}$, which has exceptionally strong pinning, $J_{c}$ is of order $0.1 J_{d}$ [31], a value now being approached by YBCO-coated conductors under certain circumstances. However, it is always worth questioning whether the active cross section carrying current differs from the whole cross section, especially with HTS conductors and perhaps also with $\mathrm{MgB}_{2}$ [9], [32], [33].

\section{Niobium-TitaniUm AlLOY}

$\mathrm{Nb}-\mathrm{Ti}$ alloy superconductors have been the "workhorse" materials of the superconductor industry for the past 40 years [1]. They were discovered in the 1960s to have a high upper critical field ( $11 \mathrm{~T}$ at $4.2 \mathrm{~K}$ and $14 \mathrm{~T}$ at $2 \mathrm{~K})$, to codraw well with $\mathrm{Cu}$ and to have good ductility. Early magnets performed poorly due to premature quenching, which was not understood at the time. However, the importance of subdividing the superconductor in order to provide 
intrinsic stability against flux jumps was recognized in the late 1960s [16]. This discovery, together with the discovery by the Rutherford group [34] that twisting the wire would reduce filament coupling, led to wires with greatly improved properties. Several major milestones were achieved in the 1980s using $\mathrm{Nb}-\mathrm{Ti}$, including the first superconducting accelerator, the Tevatron, in 1983, and the first large-scale superconducting commercial application, MRI, in the early 1980s.

The properties of $\mathrm{Nb}-\mathrm{Ti}$ alloy superconductors improved slowly throughout the 1960 s and early 1970s, so that by the time conductor was ordered for the Tevatron, a critical current specification of $1800 \mathrm{~A} / \mathrm{mm}^{2}(5 \mathrm{~T}, 4.2 \mathrm{~K})$ could be achieved. Although a recipe for improving $J_{c}$ had been developed along empirical lines by industrial manufacturers, the technology lacked a good fundamental understanding. In the early 1980s, a collaboration of university, national laboratory and industrial partners, led by the University of Wisconsin Applied Superconductivity Center, was formed to improve the understanding of flux pinning in $\mathrm{Nb}-\mathrm{Ti}$ and to incorporate this improved understanding into the manufacturing technology for $\mathrm{Nb}-\mathrm{Ti}$ [35]. The driving force at the time was the high performance requirements for the Superconducting Super Collider, and much of the work was sponsored by the Office of High Energy Physics of the U.S. Department of Energy (DOE). The steps taken to improve $\mathrm{Nb}-\mathrm{Ti}$ performance can be divided into two categories - extrinsic and intrinsic, and an apt description of the process of systematic improvement, coined at one of the LTS workshops, is "peeling the onion."

First, several extrinsic limitations were discovered and corrected. These include the formation of intermetallics at the $\mathrm{Cu} / \mathrm{Nb}-\mathrm{Ti}$ interface during the precipitation heat treatment steps [36]. This was corrected by the use of a $\mathrm{Nb}$ diffusion barrier wrapped around the $\mathrm{Nb}$-Ti filaments during billet construction [37]. Another problem is the tendency of the filaments to undergo distortion during fabrication, called "sausaging." Sausaging is caused by the difference in mechanical properties between the $\mathrm{Nb}-\mathrm{Ti}$ and $\mathrm{Cu}$ matrix, and can be prevented by placing the filaments in a dense stack in the $\mathrm{Cu}$ matrix, rather than having them widely spaced [38]. Adjusting the placement and temperature of the multiple heat treatments required to achieve a high $J_{c}$ can also help reduce the differences in mechanical properties between the $\mathrm{Nb}-\mathrm{Ti}$ filaments and the $\mathrm{Cu}$ matrix.

The first intrinsic limitation that was found and corrected was the inhomogeneity of the $\mathrm{Nb}-\mathrm{Ti}$ alloy, caused by a distribution of Ti-rich regions [39]. These responded very differently to the precipitation heat treatment and, thus, prevented establishing an optimum heat treatment to yield a uniform and predictable distribution of alpha-Ti precipitates necessary for effective flux pinning [40]. As a result of the improved understanding of $\mathrm{Nb}-\mathrm{Ti}$ alloys, the current density was increased by about $100 \%$, to $>3700 \mathrm{~A} / \mathrm{mm}^{2}$ at $5 \mathrm{~T}, 4.2 \mathrm{~K}$ [41].

Multifilamentary Nb-Ti fabricated by conventional technology is a mature field, and the rapid improvements seen from 1983 to 1990 have slowed. However, a new approach to

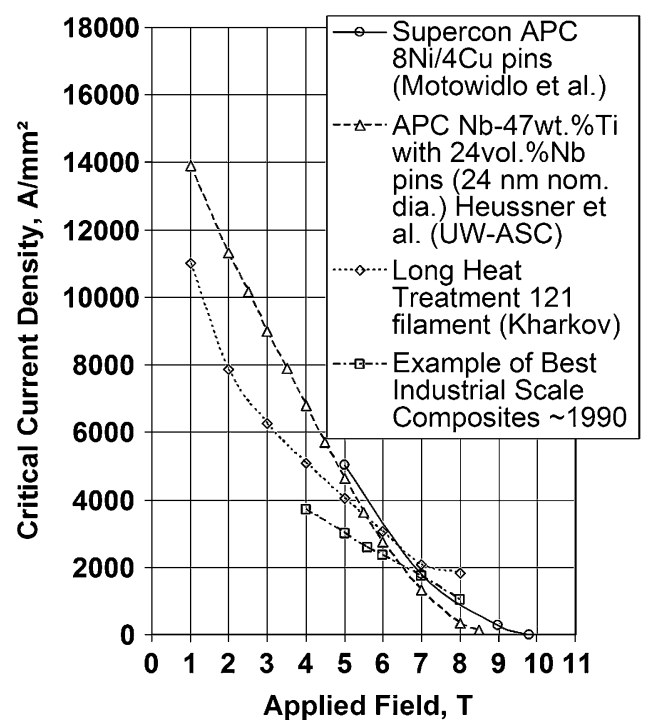

Fig. 4. Critical current density of conventional and artificial pinning center composites, showing that it is possible to further enhance the current density of $\mathrm{Nb}-\mathrm{Ti}$ by tuning the pinning center properties. Data is sourced from [41], [43], and [45].

the fabrication of $\mathrm{Nb}-\mathrm{Ti}$ using "artificial" or engineered pinning centers (APCs) was suggested in 1985 and continues to enable progress [42], [43]. The basic idea is to incorporate a normal metal into the $\mathrm{Nb}-\mathrm{Ti}$ at the beginning of the fabrication process and then to coreduce both $\mathrm{Nb}-\mathrm{Ti}$ and the pinning center material by extrusion and drawing until the normal metal component has the correct size and spacing for optimum vortex pinning. The original approach was to drill holes in a $\mathrm{Nb}-\mathrm{Ti}$ ingot and load metal rods that are compatible with the $\mathrm{Nb}-\mathrm{Ti}$ (e.g., $\mathrm{Nb}$ ). However, a more practical method from the manufacturing standpoint is to assemble a jellyroll of alternating $\mathrm{Nb}-\mathrm{Ti}$ and $\mathrm{Nb}$ sheets. This jellyroll is loaded into a $\mathrm{Cu}$ can, compacted, and extruded to produce a "clad monofilament" element, which is cut into short lengths, loaded into the final $\mathrm{Cu}$ can, and extruded. The resulting multifilamentary composite is then drawn to final wire size. Using this approach, long lengths of $\mathrm{Nb}-\mathrm{Ti}$ superconductor have been produced with $J_{c}$ values exceeding those obtained for conventionally processed $\mathrm{Nb}-\mathrm{Ti}$, in the field range from 2-7 T. Initially, the high field (6-8 T) performance was not as good for the APC wires; however, recent results with ferromagnetic pinning centers in place of the $\mathrm{Nb}$ pinning centers have shown excellent properties at higher fields as well [43], [44]. The best $J_{c}$ values for both conventional and APC $\mathrm{Nb}-\mathrm{Ti}$ are shown in Fig. 4. In this graph, we also show some recent optimization using very long heat treatment times by the Khar'kov group that have driven the $\mathrm{Nb}-\mathrm{Ti}$ critical current density to over $4000 \mathrm{~A} / \mathrm{mm}^{2}$ at $5 \mathrm{~T}, 4.2 \mathrm{~K}$ [45].

The present rate of production for $\mathrm{Nb}-\mathrm{Ti}$ superconductor is over 200 tons/year, with one project, the Large Hadron Collider (LHC) of Consel European pour la Recherche Nucleaire (CERN), accounting for about half of the total. The LHC superconductor procurement will be complete in 2006. It is unlikely that the growth rate of MRI systems will be adequate to make up for this lost LHC volume, and the next large international fusion magnet project, International 
Tokomak Experimental Reactor (ITER), will utilize mostly $\mathrm{Nb}_{3} \mathrm{Sn}$. However, much of the existing $\mathrm{Nb}-\mathrm{Ti}$ production capacity and equipment will very likely be needed for the ITER $\mathrm{Nb}_{3} \mathrm{Sn}$ procurements (see below). Thus, while the LTS industry is expected to undergo a transition, overall production volume is expected to remain steady.

\section{NiOBIUM-TIN}

$\mathrm{Nb}_{3} \mathrm{Sn}$ in a prototype wire form was the first material to show the possibility of high-field superconductivity in 1961; its $H_{c 2}$ reaches $\sim 30 \mathrm{~T}$ at $2 \mathrm{~K}$, substantially exceeding $\mathrm{Nb}-\mathrm{Ti}$ and making it the prime candidate for higher magnetic field systems [1]. Progress was initially limited, primarily due to the fact that $\mathrm{Nb}_{3} \mathrm{Sn}$ is a brittle intermetallic compound which could only be made as a tape, while $\mathrm{Nb}-\mathrm{Ti}$ is a ductile alloy, enabling very flexible architectures. However, starting in the early 1970s, practical $\mathrm{Nb}_{3} \mathrm{Sn}$ multifilamentary conductors were successfully developed with the bronze route and many laboratory and high field NMR magnets were and are still being made. As applications moved to fields exceeding the practical operating field of $\mathrm{Nb}-\mathrm{Ti}$ (about $9 \mathrm{~T}$ at $4.2 \mathrm{~K}$ and $12 \mathrm{~T}$ at $1.8 \mathrm{~K}$ ), there was strong interest in $\mathrm{Nb}_{3} \mathrm{Sn}$, driven also by plasma containment applications.

There are several processes for fabricating multifilamentary $\mathrm{Nb}_{3} \mathrm{Sn}$ wires that permit processing ductile precursors down to final wire size, followed by reaction of the components to form the $\mathrm{Nb}_{3} \mathrm{Sn}$ intermetallic compound. The so-called bronze process involves codrawing $\mathrm{Nb}$ rods in a matrix of $\mathrm{Cu}-\mathrm{Sn}$ alloy, which is made as $\mathrm{Sn}$-rich as practical, while still maintaining good ductility (around $15 \mathrm{wt} \%$ $\mathrm{Sn})$. However, much residual $\mathrm{Cu}$ is left behind after reaction, making $A_{\mathrm{sc}}$ a small $(\sim 0.2)$ fraction of the conductor package. To obtain a higher volume fraction of $\mathrm{Nb}_{3} \mathrm{Sn}$ and, hence, higher overall $J_{c}$ values, the internal Sn process was introduced. This multifilamentary composite contains $\mathrm{Nb}$ filaments in a $\mathrm{Cu}$ matrix and the $\mathrm{Sn}$ is introduced as a separate component, usually as a core added to the $\mathrm{Nb}-\mathrm{Cu}$ multifilamentary composite. These two processes, together with the newer powder-in-tube (PIT) process, are illustrated in Fig. 5.

Recently, the two principal technology drivers for $\mathrm{Nb}_{3} \mathrm{Sn}$ have been magnets for high-energy physics (HEP) accelerators and for magnetic fusion energy (MFE); a third, though less publicly discussed one, has been very high field NMR magnets used for the 800- and 900-MHz class spectrometers. In the early 1990s, both HEP and MFE initiated programs to develop superconductors and magnets for high field $(>12 \mathrm{~T})$ applications. The focus of the HEP effort was dipole and quadrupole magnets, while the fusion program focus was solenoidal and toroidal field coils for ITER [46]. Both programs chose $\mathrm{Nb}_{3} \mathrm{Sn}$ as the most promising conductor for high fields, and conductor development programs were initiated in industry. During the 1990s, the ITER program was the primary customer for $\mathrm{Nb}_{3} \mathrm{Sn}$, ordering more than 20 tons of wire from companies in Europe, Japan, Russia, and the United States. This program helped establish a large-scale

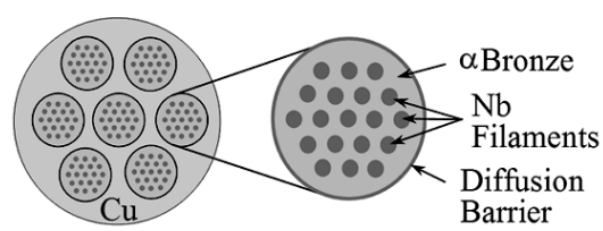

Bronze Process

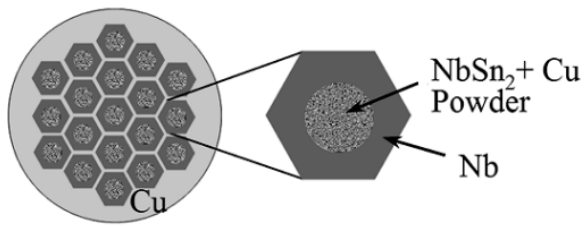

PIT - Powder in Tube

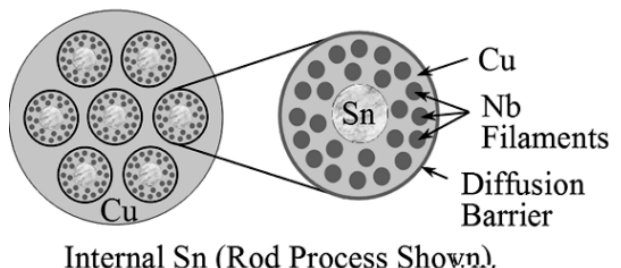

Fig. 5. Bronze, PIT, and internal Sn conductor processes for fabricating $\mathrm{Nb}_{3} \mathrm{Sn}$. (Figure courtesy of M. Naus, University of Wisconsin, Madison).

production capability for $\mathrm{Nb}_{3} \mathrm{Sn}$, and stimulated further development of bronze, internal Sn, and PIT multifilamentary wire fabrication techniques [47], [48].

As a result of the accelerator dipole design efforts at the U.S. high energy physics laboratories, it became clear that higher field, cost-effective magnets required higher current density and reduced manufacturing costs. Thus, in 1999, DOE initiated a new conductor development program for $\mathrm{Nb}_{3} \mathrm{Sn}$ [49], with the goal of providing a cost-effective, high-performance superconductor for next-generation high energy physics colliders, as well as upgrades for existing colliders at Fermi National Accelerator Laboratory, Batavia, IL, and CERN. The target conductor specifications are listed in Table 1. The emphasis is on $\mathrm{Nb}_{3} \mathrm{Sn}$ made by industrial partners with large-scale $\mathrm{Nb}_{3} \mathrm{Sn}$ production experience. Improvements in the critical current density $J_{c}$ in the noncopper part of the wire has been the highest priority, followed by reduction in the effective filament diameter $d_{\text {eff }}$, and then by lower cost.

The first-priority goal of the program, achieving an increase in the noncopper $J_{c}(12 \mathrm{~T})$ from 2000 to $3000 \mathrm{~A} / \mathrm{mm}^{2}$, was reached in 2002 [50]. This goal was achieved without an increase in cost per kilogram, so the cost-performance ratio has decreased significantly (Fig. 6). This new wire not only has excellent $J_{c}$ values, but can be produced in long lengths as well. In 2003, Oxford Superconducting Technology delivered $100 \mathrm{~kg}$ of this wire for use in a new dipole magnet at Lawrence Berkeley National Laboratory (LBNL), Berkeley, $\mathrm{CA}$, that recently reached a new dipole field record of nearly $16 \mathrm{~T}$ [51].

Development of magnet designs that maintain a relatively low transverse stress on the conductor in the highest field regions, coupled with improvements in $\mathrm{Nb}_{3} \mathrm{Sn} J_{c}$ values, 


$$
\begin{aligned}
& \mathrm{J}_{\mathrm{c}} \text { (non-copper, 12T): } 3000 \mathrm{~A} / \mathrm{mm}^{2} \\
& \mathrm{~J}_{\mathrm{e}}(12 \mathrm{~T}):>1000 \mathrm{~A} / \mathrm{mm}^{2} \\
& \text { Effective filament size: }<40 \mu \mathrm{m}
\end{aligned}
$$

Process unit size: scaleable to $>100 \mathrm{~kg}$ and average piece lengths $>10,000 \mathrm{~m}$ in wire

diameters of $0.3 \mathrm{~mm}$ to $1.0 \mathrm{~mm}$

Wire cost: $<\$ 1.50 / \mathrm{kA}-\mathrm{m}(12 \mathrm{~T}, 4.2 \mathrm{~K})$

Short heat treatment times: maximum $400 \mathrm{hrs;}$ target $50 \mathrm{hrs}$ for wind and react magnets

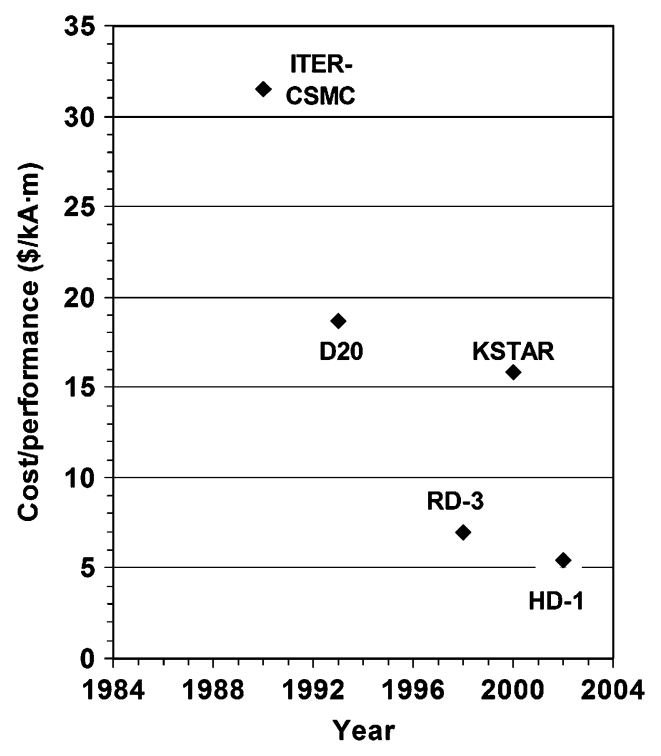

Fig. 6. Cost/performance parameters for filamentary $\mathrm{Nb}_{3} \mathrm{Sn}$ conductors used for tokamak plasma containment devices (ITER and KSTAR) and for high-field accelerator dipoles (D-20, RD-3, and HD-1). The trends show that cost has come down markedly with time, partly because of production experience and partly because $J_{c}$ has markedly increased. $J_{c}(12 \mathrm{~T}, 4.2 \mathrm{~K})$ of conductor for HD-1 is about four times that for ITER.

means that $\mathrm{Nb}_{3} \mathrm{Sn}$ has the technical performance and cost-effectiveness for use in dipole magnets up to at least $16 \mathrm{~T}$, and perhaps up to 18 or $20 \mathrm{~T}$. Detailed magnet design efforts are underway at several HEP laboratories to determine how far $\mathrm{Nb}_{3} \mathrm{Sn}$ can be pushed, and for the selection of the best conductor design for such fields.

After completion of the first phase of ITER, that project is moving ahead with the next phase, which is an international tokamak test facility [46]. The ITER design requires the production of an enormous quantity of $\mathrm{Nb}_{3} \mathrm{Sn}$ conductor (approximately $500 \mathrm{t}$ over $3-4$ years). The main challenge will be to scale up the yearly production level from the present $10 \mathrm{t} /$ manufacturer to the required $150 \mathrm{t} /$ manufacturer.

The largest present commercial application of $\mathrm{Nb}_{3} \mathrm{Sn}$ is in the area of NMR magnets. This application is growing rapidly and moving steadily to higher fields. Recent improvements in $J_{e}$ of $\mathrm{Nb}_{3} \mathrm{Sn}$ have extended its useful field range to at least $21 \mathrm{~T}$ at $1.8 \mathrm{~K}$, making persistent mode $900-\mathrm{MHz}$ systems possible. Significant challenges had to be overcome in order to achieve these high fields in a practical NMR magnet. Since this is a very competitive commercial area, magnet construction details are not usually published. Oxford/Varian and Bruker both have working $900-\mathrm{MHz}$ systems with an operating field of $21.1 \mathrm{~T}$ and very low field drift rates. The increased performance of recent $\mathrm{Nb}_{3} \mathrm{Sn}$ conductors for HEP use at 10-16 $\mathrm{T}$ has also benefited very high field applications, because the upper critical field was increased [52]. It is now widely expected that the newer $\mathrm{Nb}_{3} \mathrm{Sn}$ conductors will permit 1-GHz NMR magnets to be made of $\mathrm{Nb}-\mathrm{Ti}$ and $\mathrm{Nb}_{3} \mathrm{Sn}$. This would require persistent operation at a field of about 23.8 T in a material with an upper critical field of about 28-29 $\mathrm{T}$ at the operating temperature, a truly challenging but attainable goal.

\section{Niobium-ALUMinUm}

The driver for interest in another A-15 compound $\mathrm{Nb}_{3} \mathrm{Al}$ is an even higher magnetic field capability than $\mathrm{Nb}_{3} \mathrm{Sn}$. Two approaches for fabricating $\mathrm{Nb}_{3} \mathrm{Al}$ are being developed. The first is analogous to the internal tin process for fabricating $\mathrm{Nb}_{3} \mathrm{Sn}$. A composite of $\mathrm{Nb}$ and $\mathrm{Al}$ is formed, most often using a jelly roll technique to make the composite subelement which is extruded. This step is followed by drawing, hexing, and restacking into the final composite with a $\mathrm{Cu}$ matrix, which is then drawn to final size and reacted to form $\mathrm{Nb}_{3}$ Al. Sumitomo Electric Industries, Osaka, Japan, has used this approach to manufacture several tons of wire for a model coil for the ITER project; so manufacturability has been demonstrated [53]. However, the $\mathrm{Nb}_{3} \mathrm{Al}$ layer formed by this diffusion step is off-stoichiometry, and both $T_{c}$ and $H_{c 2}$ are suppressed. This in turn leads to a $J_{c}$ versus field performance inferior to $\mathrm{Nb}_{3} \mathrm{Sn}$.

An alternate processing route has been developed by the former National Research Institute for Metals (NRIM), now the National Institute for Materials Science (NIMS) Tsukuba, Japan, in Japan. In this process, a composite of $\mathrm{Nb}$ and $\mathrm{Al}$ is assembled and drawn to produce a fine-scale composite where the $\mathrm{Nb}$ and $\mathrm{Al}$ components have 100-200-nm dimensions. This composite is heated rapidly to $1900{ }^{\circ} \mathrm{C}$ and then quenched to produce a body-centered cubic solid solution alloy with the stoichiometric $\mathrm{Nb}_{3} \mathrm{Al}$ composition. Finally, the composite is heated to about $800{ }^{\circ} \mathrm{C}$, where the A15 phase is formed, with high $T_{c}, H_{c 2}$, and good 
high-field $J_{c}$ characteristics. By 1996, the NRIM team scaled up the rapid heat/quench step to produce relatively long lengths using a reel-to-reel system where the wire was resistively heated and then quenched in liquid Ga [54]. Ongoing optimization of this process has steadily raised $T_{c}$, $J_{c}$, and $H_{c 2}$ of rapidly quenched $\mathrm{Nb}_{3} \mathrm{Al}$ [54], [55]. However, manufacture of the precursor remains challenging, as does the production quenching, addition of stabilization, and achieving properties superior to the most recent $\mathrm{Nb}_{3} \mathrm{Sn}$. The NIMS group remains very active in pursuing the possibilities of the rapid quench technique and have seeded additional studies on this important material [56].

Chevrel phases, with intermediate transition temperatures, were also actively developed for superconducting wire [1], but this effort has now been largely abandoned.

\section{BSCCO-2212}

The HTS BSCCO-2212, with a $T_{c}$ of around $90 \mathrm{~K}$, has been eclipsed for most high-temperature applications by BSCCO-2223, which has a higher $T_{c}$ and a higher irreversibility field (see next section). However, BSCCO-2212 has superior $J_{c}$ at low temperatures (below about $20 \mathrm{~K}$ ) and high fields. For example, using their preannealing intermediate rolling (PAIR) process on long length dip-coated tapes, Showa Electric Wire and Cable Co. Ltd., Kawasaki, Japan, in collaboration with NRIM (NIMS) and the Japan Science and Technology Corporation, Tokyo, Japan, have achieved $J_{c}$ of $7100 \mathrm{~A} / \mathrm{mm}^{2}$ in self-field and $3500 \mathrm{~A} / \mathrm{mm}^{2}$ in $10 \mathrm{~T}$ parallel to the tape plane [56]. This has enabled application in high-field inserts for NMR and other applications [57], [58]. At present, BSCCO-2212 holds the record for producing the highest field with a superconducting magnet. The BSCCO-2212 tape, produced by Oxford Superconducting Technology (OST), Carteret, NJ, and wound into an insert magnet at the U.S. National High Magnetic Field Laboratory (NHMFL), Tallahassee, FL, achieved a 5-T increase in field in a background field of $20 \mathrm{~T}$ from a copper Bitter coil [8].

Also, BSCCO-2212 has been fabricated as a round wire in a silver matrix [Fig. 1(c)] with excellent critical current- $J_{e}$ at $20 \mathrm{~T}, 4.2 \mathrm{~K}$ over $500 \mathrm{~A} / \mathrm{mm}^{2}$ [59]. This wire can be used as a direct substitute for LTS wires in Rutherford-type cables [60] in applications such as accelerator magnets, and the first series of prototype coils have been made [61]. Two issues must be resolved for this application to proceed. First, the cost/performance ratio must be reduced from the roughly $\$ 50 / \mathrm{kAm}$ value at present, to under $\$ 10 / \mathrm{kAm}$. Second, practical methods for achieving stringent heat treatment control in an industrial environment must be developed. A partial melting step is required to achieve optimum properties, and the temperature must be controlled to within a few degrees at around $870{ }^{\circ} \mathrm{C}$. At present, three companies worldwide are manufacturing BSCCO-2212-Showa, Nexans, and OST. All companies have demonstrated excellent $J_{c}$ values. Showa and Nexans, Jeumont, France, [62] have both demonstrated that they can make 1600-m lengths of wire without breakage and can control the heat treatment to achieve uniform properties.

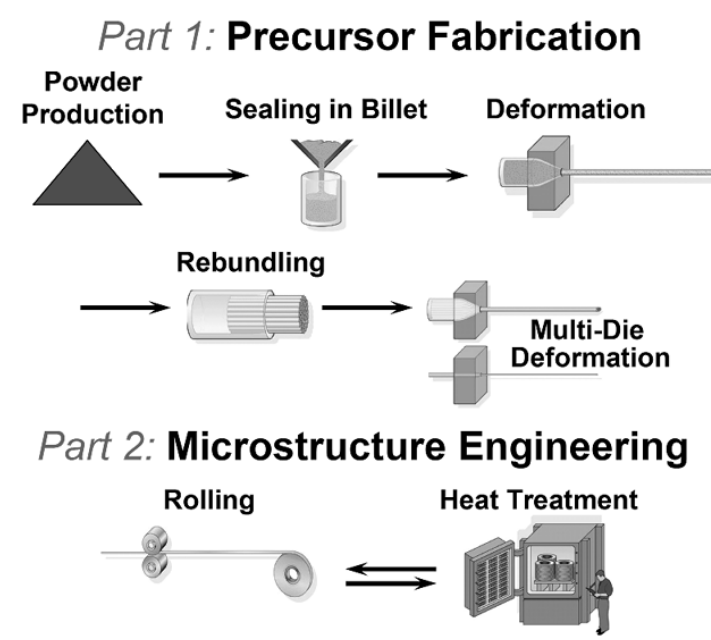

Fig. 7. Schematic of the OPIT process for making Bi-2223 superconducting wire such as that shown in Fig. 1(d). Starting powder based on Bi-2212 and required $\mathrm{Ca}-\mathrm{Cu}-\mathrm{O}$ balancing ingredients is packed in a silver billet, deformed to a monofilament stack and then rebundled into a multifilament stack. The heat treatment which drives the 2212 to 2223 phase conversion occurs in two or more heat treatments separated by an intermediate rolling step which helps densify the tape.

\section{BSCCO-2223}

The HTS $(\mathrm{Bi}, \mathrm{Pb})_{2} \mathrm{Sr}_{2} \mathrm{Ca}_{2} \mathrm{Cu}_{3} \mathrm{O}_{10}$, called BSCCO-2223, with a $T_{c}$ of about $110 \mathrm{~K}$, is used in the first generation of commercial HTS conductor, a composite of the superconductor with silver [3]. As shown in the cross section in Fig. 1, this first-generation HTS wire has a tape shape, typically 0.2 by $4 \mathrm{~mm}$, consisting of 55 or more tape-shaped filaments, each $10 \mu \mathrm{m}$ thick and up to $200 \mu \mathrm{m}$ wide, embedded in a silver alloy matrix. The filaments consist of grains of BSCCO-2223, up to $20 \mu \mathrm{m}$ long, often arranged in colonies sharing a common $c$ axis. For some applications, the wire is laminated to stainless steel tapes on either side for enhanced mechanical properties and environmental protection. This kind of HTS wire is manufactured by a number of companies, including American Superconductor Corporation (Devens, MA), European Advanced Superconductor GmbH (formerly Vacuumschmelze, Hanau, Germany), Innova Superconductor Technology (Beijing, China), Sumitomo Electric Industries Ltd. (Japan), and Trithor GmbH (Rheinbach, Germany). Worldwide capacity exceeds $1000 \mathrm{~km}$ per year.

While precise details of the industrial production process are not public, the basic PIT deformation process is schematized in Fig. 7 [3], [10]. A powder consisting of a mixture of $\mathrm{Pb}$-containing BSCCO-2212, alkaline earth cuprates, copper oxide, and other oxides is initially prepared by an aerosol, spray-dry or simple oxide mixing process; the overall cation stoichiometry is chosen to match that of BSCCO-2223. The powder is packed into a silver tube, which is sealed, evacuated, and drawn through a series of dies, elongating it into a hexagonal rod. These rods are next cut, assembled into a multifilament bundle (55-85 filaments are typical), and inserted into a second silver or silver alloy tube. This tube is sealed, evacuated, and further drawn into a fine round wire, 


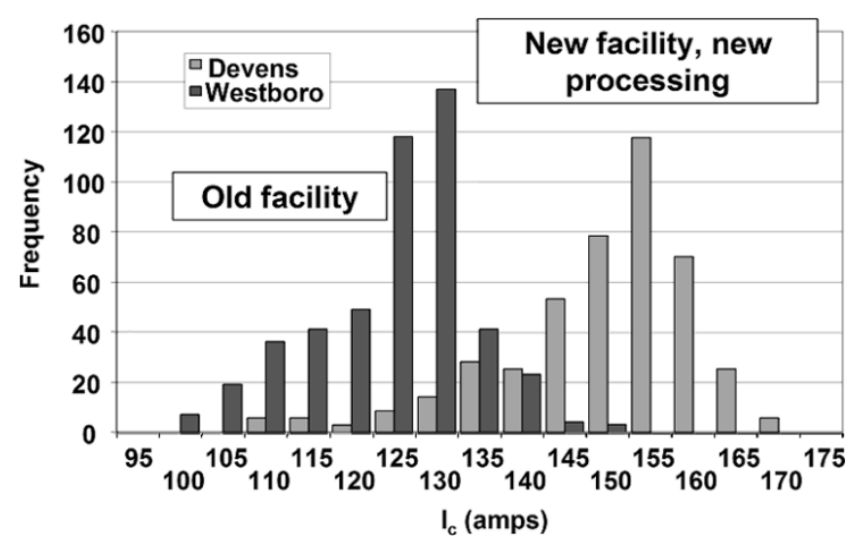

Fig. 8. The $I_{c}(77 \mathrm{~K}$, self-field) distribution from production runs of recent AMSC unlaminated first-generation HTS wires based on BSCCO-2223, with $4.2 \times 0.21 \mathrm{~mm}^{2}$ cross section and $>150 \mathrm{~m}$ length (courtesy of AMSC). The 170-A result corresponds to an engineering critical current density of over $190 \mathrm{~A} / \mathrm{mm}^{2}$.

which is subsequently deformed to a tape shape in a rolling mill, in multiple steps of rolling and heat treatment to texture and react the powder inside the wire to form BSCCO-2223. The superconducting fill factor is typically $30 \%-40 \%$.

Three important insights into this process are as follows. First, the use of silver, a relatively expensive metal, is necessary because silver, like other noble metals, is inert to the superconductor, and particularly because it permits rapid oxygen diffusion at high temperatures without itself oxidizing. This latter property allows control of the oxygen activity in the reaction of the powder to BSCCO-2223. The silver also acts as an electrical and mechanical stabilizer in intimate contact with the superconducting filaments. Second, the use of the rolling process to flatten the wire to a tape shape is necessitated by the need to texture the powder, which consists of mica-like grains of BSCCO-2212, along with the less aspected other oxides. High texture is the key to a high current density in the final product. Third, the success of this process with BSCCO rather than other HTS materials appears to be related to easy slip along the weakly bonded double- $\mathrm{Bi}, \mathrm{Pb}$-oxide layers; this facilitates texturing during the rolling deformation. The easy slip plane also enables formation of colonies or stacks of many highly aspected grains rotated in apparently random orientations with respect to each other around a common $c$ axis perpendicular to the $\mathrm{CuO}$ planes. This structure is believed to facilitate current flow as discussed in Section III above [27].

$J_{c}$ of BSCCO-2223-based wire has been a research and development focus worldwide for 15 years, increasing steadily over time, with long-length $(>150 \mathrm{~m})$ wires now achieving up to a maximum of $170 \mathrm{~A}$ and an average above $150 \mathrm{~A}$ in end-to-end $I_{c}$ at $77 \mathrm{~K}$ and self-field in $0.21 \times 4.2 \mathrm{~mm}$ tape (Fig. 8) [3], [10]. The maximum $I_{c}$ corresponds to an engineering critical current density of close to $180 \mathrm{~A} / \mathrm{mm}^{2}$. Given a $40 \%$ superconductor fill factor in the wire, this corresponds to $J_{c}$ of $450 \mathrm{~A} / \mathrm{mm}^{2}$. At a temperature of $30 \mathrm{~K}$ and $2 \mathrm{~T}$, of interest, for example, in rotating machinery applications, the current density is enhanced over its $77 \mathrm{~K}$ self-field value by almost a factor of two. These

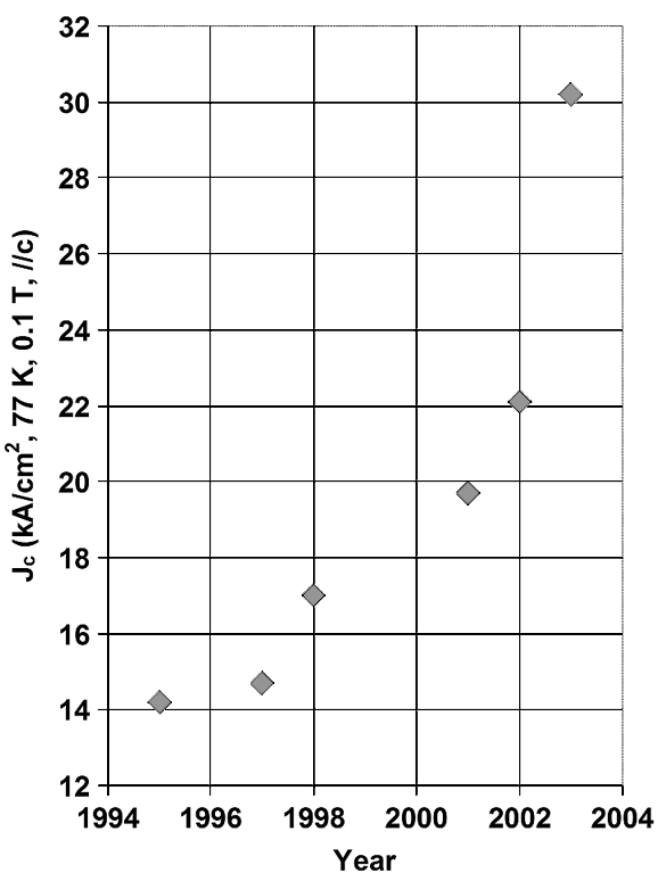

Fig. 9. Steady progress in understanding the current limiting mechanisms in BSCCO-2223 first-generation HTS wires has led to continued progress in raising the current density expressed at the benchmark field of $0.1 \mathrm{~T}$ at $77 \mathrm{~K}$, at which field the tapes are always fully penetrated by the external field and self-field suppression of the $J_{c}$ is negligible. The highest value sample plotted above has a strongly self-field limited critical current of $182 \mathrm{~A}(77 \mathrm{~K})$ in zero-applied field, while its estimated self-field-free $I_{c}$ is $235 \mathrm{~A}$, corresponding to $J_{c}$ of $266 \mathrm{~A} / \mathrm{mm}^{2}$. (Courtesy of AMSC and the University of Wisconsin, Madison.)

performance levels have been applied successfully in a variety of commercial-level prototypes and appear adequate for most power applications. At this point, the main value of further increasing $I_{c}$ is reducing the price-performance ratio $\$ / \mathrm{kAm}$. First-generation HTS wires are currently sold at \$150-200/kAm, and with further manufacturing efficiencies, price-performance of $\$ 50 / \mathrm{kAm}$ is expected, even without further increase in $I_{c}$.

More progress in $I_{c}$ seems feasible, based on an improved understanding of the materials science of BSCCO-2223 and its phase conversion, closely correlated to knowledge of where the current flows and how it is limited by microstructural defects [63]. This task has been pursued by the DOE-supported Wire Development Group, an industry-national lab-university partnership, during the past dozen years. Recently there has been progress in mitigating two important current-limiting mechanisms. One is residual porosity left over from the dedensification that occurs during the reaction of 2212 phase to 2223 and the cracking that occurs during the intermediate rolling step that is required to remove such porosity. The second is due to unreacted streaks of 2212 phase that occur either as intergrowths within the 2223 phase or as individual grains within a larger colony of $\sim 102223$ grains which share a common $c$ axis. Porosity and cracking have been remarkably reduced by processing at hydrostatic pressures of up to $150 \mathrm{bar},[63]-[66]$ advancing $J_{c}$ at the appropriate benchmark of $0.1 \mathrm{~T}$ at $77 \mathrm{~K}$ to $304 \mathrm{~A} / \mathrm{mm}^{2}$, as shown in Fig. 9. The corresponding zero-field value at 

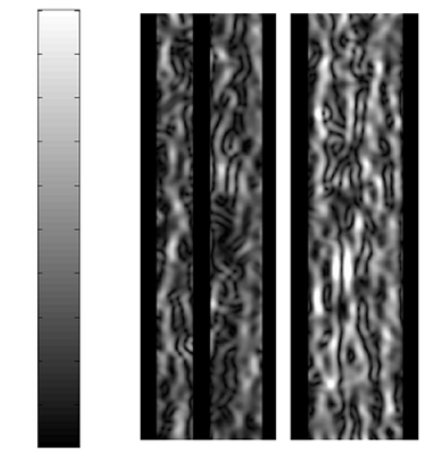

$\mathrm{J}_{\mathrm{c}}=0$

Fig. 10. Current distribution reconstructions from magnetooptical images at $77 \mathrm{~K}$ and $40-\mathrm{mT}$ field parallel to the $a b$ planes of two monofilament AMSC BSCCO-2223 first-generation HTS wires. This imaging condition develops a current density that is within $5 \%$ of the self-field current density under normal self-field test conditions. The $J_{c}(77 \mathrm{~K}$, self-field) values for the $1 \mathrm{~atm}$-processed tape was $390 \mathrm{~A} / \mathrm{mm}^{2}$ and that for the 148 bar-processed (overpressure-OP) tape (right-hand pair) was $480 \mathrm{~A} / \mathrm{mm}^{2}$. In the left-hand pair, the brightest areas are $500 \mathrm{~A} / \mathrm{mm}^{2}$ but $2000 \mathrm{~A} / \mathrm{mm}^{2}$ in the right pair. The many bright areas in the right-hand picture show that many regions exceed $2000 \mathrm{~A} / \mathrm{mm}^{2}$. (The black bar in the $1 \mathrm{~atm}$-processed tape is a nonmeasurable area in the magnetooptical image.) Data taken from [63].

$77 \mathrm{~K}$ has surpassed $200 \mathrm{~A}$ in a $4.2 \times 0.21 \mathrm{~mm}^{2}$ wire. This marks a doubling of $J_{c}$ in the last five years, and the pace is accelerating as fundamental understanding grows. Similarly, improved heat treatments have reduced the amount of residual 2212 phase. Recent magnetooptical current reconstructions (Fig. 10) on a high- $J_{c}$ monofilament conductor with $J_{c}(77 \mathrm{~K}, \mathrm{sf})=350 \mathrm{~A} / \mathrm{mm}^{2}$ [63] show that there is still great headroom in the system. Remarkably, $J_{c}$ locally reaches well over $2500 \mathrm{~A} / \mathrm{mm}^{2}$, five times higher than its average value, and regions of $J_{c}$ exceeding $1000 \mathrm{~A} / \mathrm{mm}^{2}$ are 50 to $100 \mu \mathrm{m}$ long, several times the $\sim 20 \mu \mathrm{m}$ grain length. Although there is a tendency for the highest $J_{c}$ regions to be located at the $\mathrm{Ag}$-superconductor interface, in fact, very high $J_{c}$ regions are located throughout the filament. The $J_{c}$ distribution is both higher and spatially larger in the overpressure processed tapes, consistent with strong reduction of porosity and cracking.

With the strong performance improvement, cost reduction, and successful manufacturing scale-up, first-generation BSCCO-2223-based HTS wire has proven successful in meeting applications requirements and is being widely used in commercial-level prototypes. No other HTS wire technology is likely to replace it for at least several years [10]. It will be the basis of the first commercial HTS applications, which are expected to be largely in the electric power arena and in magnets where operation at temperatures above $30 \mathrm{~K}$ is preferred. The longer term future for this wire depends on the degree to which its price-performance ratio can be reduced and on the success of alternatives like second-generation HTS wire based on YBCO-coated conductor.

\section{YBCO-COATED CONDUCTORS}

The vision for a second generation of HTS wire is based on a quasi-single-crystal layer of $\mathrm{YBa}_{2} \mathrm{Cu}_{3} \mathrm{O}_{7}$, with grain-to-grain misorientations of order $5^{\circ}$ or less to enable high current by exploiting the strong grain-to-grain coupling which occurs under these conditions (see Section III) [9], [67]-[74]. Such a continuous YBCO film must be manufactured over long lengths and at low cost with only a nominal $\mathrm{Ag}$ fraction [75]. After about a decade of effort, it was shown in 2003 by multiple fabrication routes that continuous processing of lengths of $10-50 \mathrm{~m}$ with high critical current properties is possible, opening the path to production scale up. Coated conductors use thin-film technology to apply an epitaxial superconductor layer to a highly biaxially textured tape-shaped template, whose texture the YBCO replicates. This is a complex, metal-supported, multifunctional, multilayer oxide structure. Cost models for coated conductors, particularly those made by nonvacuum methods, predict values below $\$ 10 / \mathrm{kAm}(77 \mathrm{~K}, 0 \mathrm{~T})$; well below the effective price-performance of copper [75]. Because of the significantly higher irreversibility field of YBCO compared to BSCCO (Fig. 2), second-generation HTS wire also offers the opportunity to achieve higher temperature operation in a given magnetic field.

All coated conductors include a flexible substrate, preferably of strong and nonmagnetic or weakly magnetic metal, typically $\sim 50 \mu \mathrm{m}$ thick, on top of which is a multifunctional oxide barrier or buffer layer, typically less than $\sim 0.5 \mu \mathrm{m}$ thick, on top of which is the superconducting YBCO layer 1-3 $\mu \mathrm{m}$ thick. A protective $\mathrm{Ag}$ layer of a few micrometers and a thicker $\mathrm{Cu}$ protection and stabilization layer complete the conductor [76]. The textured template is created by one of two basic methods, either by texturing the buffer layer by ion beam assisted deposition (IBAD) [67]-[71], [77], or inclined substrate deposition (ISD) [78], [79], or by deformation texturing the metal substrate with the rolling assisted biaxially textured substrate approach [72]-[74] and applying epitaxial oxide buffer layers (trademarked RABiTS by Oak Ridge National Laboratory, Oak Ridge, TN).

The IBAD method [67] allows for the use of strong, nonmagnetic Ni-superalloy or stainless steel substrates on which a textured IBAD layer of aligned yttria-stabilized zirconia (YSZ), gadolinium zirconate (GZO), or magnesium oxide $\mathrm{MgO}$ can be deposited [70], [77]. This process can achieve good texture (full-width at half-maximum $(\mathrm{FWHM}) \sim 10^{\circ}$ ) with a layer of YSZ that is $\sim 0.5-1 \mu \mathrm{m}$ thick. However, deposition of IBAD-YSZ is widely considered to be too slow, and, thus, too expensive, to be commercial. IBAD-MgO, however, produces excellent texture within the first $1-2 \mathrm{~nm}$, and, thus, deposition may be rapid enough to be commercially cost-effective [70], [77]. The X-ray pole-figure FWHM of recent IBAD-MgO coated conductors is much better than for IBAD-YSZ, being of order $2-4^{\circ}$ and, thus, genuinely approaching single crystal structure for the YBCO overlayer [70], although performance so far does not fully reflect this unusually high degree of texture, and achieving the required atomic level surface roughness over long lengths has proven 
challenging. The inclined substrate deposition (ISD) process is more rapid than YSZ-IBAD, since there is no resputtering during deposition, but in work to date, the texture is not as high [78], [79]. ISD may also permit simpler buffer structures. Other ion texturing processes for buffer layer deposition have also been explored [80]. All these methods of producing a textured buffer layer rely on physical vapor deposition processes, whose cost is an obstacle to achieving price-performance competitive with copper.

An advantage of the RABiTS approach is that texture can be achieved with a low-cost nonvacuum process. A strong [100] cube texture is introduced into the substrate by conventional rolling and recrystallization. Although the RABiTS approach was developed initially with pure nickel [72], substrate materials with more robust mechanical properties and reduced magnetism, notably nickel with 5 atomic \% tungsten, have now replaced pure $\mathrm{Ni}$ and achieved FWHM below $5^{\circ}[73]$.

The buffer layer is also in active development. At present, virtually all processes employ a multifunctional buffer in which the functions of seed layer for the metal-oxide interface, a metal and oxygen diffusion barrier, and cap layer lattice-matching to the YBCO interface are separated. Many such architectures are presently vying for selection. Seed layers of $\mathrm{Y}_{2} \mathrm{O}_{3}, \mathrm{Gd}_{2} \mathrm{O}_{3}$, or $\mathrm{NiO}$ have all been employed. YSZ is by far the most common Ni diffusion barrier or buffer, while $\mathrm{CeO}_{2}$ is generally employed for the cap layer interface to YBCO. IBAD-MgO buffer layers are also in rapid development.

Many different deposition methods for the crucial epitaxial YBCO layer have been developed, all achieving high critical currents in short samples. Thus, the choice among them can be driven by low cost. The leading low-cost alternatives are those based on liquid-phase chemical routes using metal-organic deposition (MOD) [75], [76] or on metal-organic-chemical vapor deposition (MOCVD) [81], though advocates of fast electron-beam deposition of either metal constituents or the so-called $\mathrm{BaF}_{2}$ route remain, as do the advocates of so-called fast pulsed laser deposition. It is also becoming increasingly recognized that the use of higher $T_{c}$ versions of the YBCO structure, for example, $\mathrm{RBa}_{2} \mathrm{Cu}_{3} \mathrm{O}_{7}$, where $\mathrm{R}$ might be $\mathrm{Eu}$ or $\mathrm{Nd}$ with a $T_{c}$ of 94-95 K could offer significant benefit to $77 \mathrm{~K}$ properties.

The design of a coated conductor is such that about $50-\mu \mathrm{m}$ substrate thickness is needed to support $1-5 \mu \mathrm{m}$ of YBCO, giving a superconductor fraction which is at best $5 \%-10 \%$ of the cross section, as compared to $30 \%-40 \%$ in the first-generation HTS wire of Fig. 1. Present short samples of both IBAD and RABiTS conductors can both exceed $2 \mathrm{MA} / \mathrm{cm}^{2}$ at $77 \mathrm{~K}$ for YBCO layers up to $\sim 2 \mu \mathrm{m}$, enabling currents of $400 \mathrm{~A} / \mathrm{cm}$ width or $160 \mathrm{~A}$ in a 4-mm-wide tape, which is comparable to first-generation wire. A significant and not well understood obstacle to further increasing $I_{c}$ is that in many cases $J_{c}$ of the YBCO layer degrades with increasing thickness. Loss of epitaxy, increasing porosity, a microstructural transition through thickness, and possibly a two-dimensional to three-dimensional transition of the vortex structure

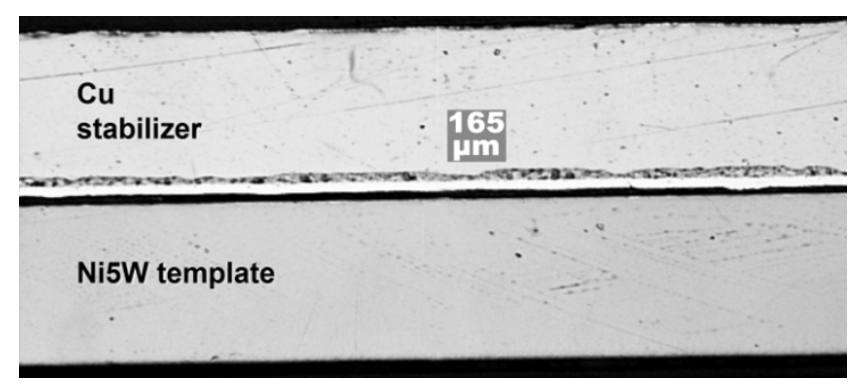

Fig. 11. Illustrative architectures [76] of a neutral axis YBCO coated conductor, showing the copper stabilizer, the solder joining the $\mathrm{Cu}$ to the YBCO, and the $\mathrm{Ni}_{5}$ at $\% \mathrm{~W}$ textured template. (Courtesy of AMSC.)

once the layer gets thicker than about $\sim 0.5 \mu \mathrm{m}$ may all play significant roles [82].

Progress on continuous reel-to-reel processes for making coated conductors has been particularly rapid in the recent year. Continuous 10-100-m lengths of 1-cm-wide conductor with end-to-end critical currents as high as $270 \mathrm{~A} / \mathrm{cm}$ width $\mathrm{A}$ at self-field at $77 \mathrm{~K}$ show that this multistep fabrication process is being mastered [83]. Uniformity along the length indicates that kilometer-length wires should be possible. Lamination of hardened copper strip to the multilayer of metal substrate, buffer, YBCO, and Ag passivation is an important step to achieve a robust, stabilized conductor. In particular, conductor designs such as those shown in Fig. 11 include a "neutral axis" conductor in which the YBCO lies at the position of zero tensile or compressive strain during bending, or a "face-to-face" conductor in which two layers of YBCO, coupled through an internal stabilizing copper layer, provide alternative current paths for each other in case of a defect in one [76].

No fundamental technical barriers to the fabrication of long-length coated conductors appear to exist, although much work remains to scale up the process to long lengths. Work will also need to focus on improving flux pinning in YBCO so as to achieve higher $J_{c}$ in field and a monotonic $J_{c}$ as a function of field angle. The introduction of these second-generation HTS wires into practical applications will be facilitated by designing them with similar size and rating as first-generation HTS wires, since all present electrical equipment design is being done with first-generation wires. Second-generation wires are also very attractive for current limiting applications because of the high electric fields that can be reached when the superconductor goes normal in response to a high fault current.

In short, second-generation HTS wires show promise for achieving a price-performance ratio below that of copper and for providing new performance features, thus significantly expanding the HTS market. Expected applications are largely in the electric power arena and for magnets where operation at temperatures above $30 \mathrm{~K}$ is preferred. Active research and development is underway at Los Alamos, Oak Ridge, and Argonne National Laboratories, at the International Superconductivity Technology Center (ISTEC), Tokyo, Japan, and at a variety of European laboratories and universities. Industrial programs are underway at American 
Superconductor Corporation and the Intermagnetics General subsidiary SuperPower Inc., Latham, NY, at Fujikura Ltd., Tokyo, Japan, Furukawa Electric Co. Ltd., Tokyo, Japan, and Sumitomo Electric Industries Ltd. in Japan, and at Theva $\mathrm{GmbH}$ in Europe. However, it should be emphasized that it will take several years to achieve a meaningful scale-up to long lengths and adequate production capacity for the commercial market.

\section{MAGNesium Diboride}

In early 2001, the Akimitsu group in Japan discovered that the long-ago-synthesized compound magnesium diboride $\mathrm{MgB}_{2}$ was a hitherto unappreciated $40 \mathrm{~K}$ superconductor [84]. Extensive work on $\mathrm{MgB}_{2}$ has now shown that it is a "conventional" s-wave superconductor but with the unconventional property that it contains two, only very weakly coupled superconducting gaps. This two-gap property is very important to its high-field performance potential [85]-[87].

A key early discovery was that randomly oriented grain boundaries in $\mathrm{MgB}_{2}$ are not obstacles to current flow, in spite of the fact that its alternating $\mathrm{Mg}$ and $\mathrm{B}$ sheet structure makes it electronically anisotropic [88]. The magnitude of this $H_{c 2}$ anisotropy is under intense study at present. In single crystals, this anisotropy is quite strongly temperature dependent and increases with decreasing temperature to values of five to seven [89]. In a "clean," very low $H_{c 2}$ limit, the zero-temperature perpendicular upper critical field $H_{c 2}^{\perp}(0)$ is only 3-4 T, while the equivalent parallel field value, $H_{c 2}^{\|}(0)$ reaches $\sim 15-17 \mathrm{~T}$. Study of dirty-limit films shows that $H_{c 2}^{\perp}(0)$ can reach more than $40 \mathrm{~T}$, while $H_{c 2}^{\|}(0)$ exceeds present measurement fields and probably is exceeds $70 \mathrm{~T}$ [90]. An important conclusion is that the $H_{c 2}(T)$ envelope of $\mathrm{MgB}_{2}$ can exceed that of $\mathrm{Nb}_{3} \mathrm{Sn}$ at any temperature, even in the weaker condition of field perpendicular to the B sheets. Because grain boundaries appear to carry current independent of misorientation, round wire conductors without texture are practical, provided the limitation of the anisotropic $H_{c 2}$ is accepted. As noted above, this anisotropy actually decreases as $H_{c 2}$ is enhanced, primarily because the lower perpendicular $H_{c 2}$ increases more rapidly than the parallel value.

$\mathrm{MgB}_{2}$ can be made by the PIT process and many groups have made prototype wires, using both prereacted (ex situ) $\mathrm{MgB}_{2}$ powder and mixtures of $\mathrm{Mg}$ and $\mathrm{B}$ powders, which must be reacted to $\mathrm{MgB}_{2}$ in situ within the wire [12]. A particularly rapid use of $\mathrm{MgB}_{2}$ wire has been for low thermal conductivity current leads in the ASTRA-2 satellite [91]. However, it is clear from analyses of the electrical resistivity of many samples, including wires, that bulk $\mathrm{MgB}_{2}$ is actually very far from being fully sintered or electrically connected [33]. Porosity and wetting by B and perhaps oxygenrich phases obstruct many grain boundaries [92], [93] and porosity is still endemic in wires [12]. Yet even so, $J_{c}$ exceeds $10^{3} \mathrm{~A} / \mathrm{mm}^{2}$ at lower temperatures and fields. In well-connected thin films $J_{c}$ values as high as $10^{5} \mathrm{~A} / \mathrm{mm}^{2}$ have been reported [94]. It, thus, appears clear that at least in the temperature range below about $25 \mathrm{~K}$, there is neither a current density, nor an upper critical field barrier to the application of $\mathrm{MgB}_{2}$. Thus, $\mathrm{MgB}_{2}$ wires could become a credible competitor to LTS-based wires or to BSCCO-based wires used in low-temperature $(<25 \mathrm{~K})$ applications. An additional quality is that the $H_{c 2}$ transition appears to be rather sharp, much more similar to low- $T_{c}$ materials [95] than to HTS materials [96]. Thus, high $n$ values and even persistent mode magnets appear feasible, making $\mathrm{MgB}_{2}$ a potential NMR or MRI magnet conductor.

Another advantage of $\mathrm{MgB}_{2}$ is that the raw material costs of both $\mathrm{B}$ and $\mathrm{Mg}$ are low; reasonable estimates suggest that even for appropriately purified material, they will be several times less than those of Nb-based superconductors [97]. Thus, the principal uncertainties of the technology are the costs and difficulties of developing the PIT composite technology or some alternative. By way of comparison, PIT technology is employed for $\mathrm{Nb}_{3} \mathrm{Sn}$ manufacture using powders of $\mathrm{NbSn}_{2}$ inside $\mathrm{Nb}$ tubes. Present manufacturing costs of $\mathrm{Nb}_{3} \mathrm{Sn}$ conductors using PIT processes are about five to six times higher than conventional metal-working approaches to $\mathrm{Nb}_{3} \mathrm{Sn}$ composites. Some of this is due to the costs of making appropriate starting powders but a large part is also due to the small scale on which PIT- $-\mathrm{Nb}_{3} \mathrm{Sn}$ is presently made. It is worth noting that the quality and $J_{c}$ of PIT- $\mathrm{Nb}_{3} \mathrm{Sn}$ composites made by ShapeMetal Innovation, Enschede, The Netherlands, are very high-conductors containing two hundred to five hundred $15-25-\mu \mathrm{m}$-diameter filaments have been made in magnet lengths [52]. Such filaments are completely decoupled, which is not at all true of the filaments in either BSCCO-2212 or -2223 multifilamentary conductors. If $\mathrm{MgB}_{2}$ is to challenge LTS, the goal must be to replicate this quality, while keeping production costs down.

In summary, $\mathrm{MgB}_{2}$ is a most intriguing new entry into the superconductor wire arena. By making $\mathrm{MgB}_{2}$ dirty, just as was done earlier when Ti was added to $\mathrm{Nb}_{3} \mathrm{Sn}, H_{c 2}$ can be greatly enhanced [86], [87], [90], [98], [99]. Indeed the two-gap nature of $\mathrm{MgB}_{2}$ adds additional versatility to the material, because the low temperature value of $H_{c 2}$ is determined by the electronic diffusivity of the dirtier band, while the initial slope of $H_{c 2}(T)$ is determined by that of the cleaner band. Carbon doping of $\mathrm{MgB}_{2}$ appears to be particularly valuable to enhancing $H_{c 2}$ [90], [98], [99]. One effective way of both enhancing $H_{c 2}$ and the flux pinning strength may be by the addition of $\mathrm{SiC}$ nanoparticles [100]. Because the fabrication of $\mathrm{MgB}_{2}$ can occur by conventional metal-working processes, the barrier to experimentation is low. The problems of fabricating $\mathrm{MgB}_{2}$ conductors commercially are being addressed by Hyper Tech Research, Columbus, OH [101], Columbus Superconductors, Genova, Italy [102], Hitachi, Hitachi City, Japan, and NIMS in Japan [103], and many other laboratories worldwide, as a good recent survey makes clear [12]. Several groups have fabricated more than $100-\mathrm{m}$ lengths of prototype wires. Assuming costs can be kept low, competition with Nb-base LTS conductors seems likely, while competition with either BSCCO-based or YBCO-based first- and second-generation HTS conductors will depend on progress in expanding the 
temperature range where high currents are maintained in several tesla fields.

\section{Conclusion}

The overall picture of superconductor wire is diverse and developing rapidly. The LTS materials $\mathrm{Nb}-\mathrm{Ti}$ and $\mathrm{Nb}_{3} \mathrm{Sn}$ are well established in high-energy physics, commercial MRI and NMR, and many low-temperature magnet applications. Their properties are well developed and their applications well understood. Even though $\mathrm{Nb}_{3} \mathrm{Sn}$ will be 50 years old this year, it has seen great progress recently. The very recently discovered $\mathrm{MgB}_{2}$ has the potential to play a growing role in these applications. BSCCO-2212 wire also has advantages for ultrahigh magnetic field use. Great changes are likely in the arena of electric power applications and magnet applications at temperatures above $30 \mathrm{~K}$. Here the HTS materials BSCCO-2223 and YBCO are the strongest candidates, with first-generation HTS wire based on BSCCO-2223 already commercially available and applied in a variety of prototypes. Indeed, the first commercial sale of HTS power equipment based on first-generation HTS wire has recently occurred for dynamic synchronous condensers supplying reactive compensation in the power grid [104]. This is a harbinger of a coming revolution in the market for superconductor wire.

\section{REFERENCES}

[1] B. Seeber, Ed., "Commercially available superconducting wires," in Handbook of Applied Superconductivity, Bristol, U.K.: Inst. of Physics, 1998, pp. 397-488.

[2] B. Seeber, Ed., "Present applications of superconductivity," in Handbook of Applied Superconductivity, Bristol, U.K.: Inst. of Physics, pp. 1165-1484.

[3] L. Masur, "Industrial high temperature superconductors: Perspectives and milestones," IEEE Trans. Appl. Superconduct., vol. 12, pp. 1145-1150, Mar. 2002.

[4] B. Seeber, Ed., "Power applications of superconductivity," in Handbook of Applied Superconductivity, Bristol, U.K.: Inst. of Phys., 1998, pp. 1485-1756.

[5] S. Kalsi et al., "Installation and operation of superconducting rotating machines," presented at the IEEE Transmission and Distribution Meeting, Dallas, TX, 2003.

[6] B. W. McConnell, M. S. Walker, and S. Mehta, "HTS transformers," IEEE Power Eng. Rev., vol. 20, pp. 7-11, June 2000.

[7] A. P. Malozemoff, J. Maguire, B. Gamble, and S. Kalsi, "Power applications of high temperature superconductors: Status and perspectives," IEEE Trans. Appl. Superconduct., vol. 11, pp. 778-781, Mar. 2002.

[8] H. W. Weijers et al., "The generation of $25.05 \mathrm{~T}$ using a $5.11 \mathrm{~T}$ $\mathrm{Bi}_{2} \mathrm{Sr}_{2} \mathrm{CaCu}_{2} \mathrm{O}_{x}$ superconducting insert magnet," Supercond. Sci. Technol., vol. 17, pp. 636-644, 2004.

[9] D. Larbalestier, A. Gurevich, D. M. Feldmann, and A. Polyanskii, "High $T_{c}$ superconducting materials for electric power applications," Nature, vol. 414, pp. 368-377, 2001.

[10] A. P. Malozemoff et al., "HTS wire: Status and prospects," Physica C, vol. 386, pp. 424-430, 2003.

[11] M. Gouge et al., Advances in Cryogenic Engineering. Melville, NY: AIP, 2004, to be published.

[12] R. Flukiger et al., "Superconducting properties of $\mathrm{MgB}_{2}$ tapes and wires," Physica C, vol. 385, pp. 286-305, 2003.

[13] (2001, May) National Energy Policy: Report of the National Energy Policy Development Group. [Online]. Available: http://www. whitehouse.gov/energy

[14] (2002, May) National Transmission Grid Study. U.S. Dept. Energy. [Online]. Available: http://tis.eh.doe.gov/ntgs/reports
[15] Grid 2030: A national vision for electricity's second 100 years (2003, July). [Online]. Available: http://www.electricity.doe.gov/ vision

[16] M. Wilson, Superconducting Magnets, Oxford, UK: Clarendon, 1983.

[17] M. Tinkham, Introduction to Superconductivity. New York, NY: McGraw Hill Inc., 1975.

[18] K. A. Mueller et al., "The development of superconductivity research in oxides," in Proc. 10th Anniversary HTS Workshop, B. Batlogg et al., Eds., 1996, pp. 3-16.

[19] A. P. Malozemoff, "Macroscopic magnetic properties of high temperature superconductors," in Physical Properties of High Temperature Superconductors, D. Ginsburg, Ed, Singapore: World Scientific, 1989, pp. 71-150.

[20] - "Flux creep in high temperature superconductors," Physica $C$, vol. 185-189, pp. 264-269, 1991.

[21] - "New developments in flux creep of high temperature superconductors," in AIP Conf. Proc., vol. 219, Y.-H. Kao et al., Eds., 1991, p. 84.

[22] R. M. Scanlan, W. A. Fietz, and E. F. Koch, "Flux pinning centers in superconducting $\mathrm{Nb}_{3}$ Sn," J. Appl. Phys., vol. 46, pp. 2244-2249, 1975.

[23] A. Gurevich and A. Pashitskii, "Current transport through low-angle grain boundaries in high-temperature superconductors," Phys. Rev. $B$, vol. 57, pp. 13878-13893, 1998.

[24] D. Dimos, P. Chaudhari, and J. Mannhart, "Superconducting transport properties in $\mathrm{YBa}_{2} \mathrm{Cu}_{3} \mathrm{O}_{7-\delta}$ bicrystals," Phys. Rev. B, vol. 41, pp. 4038-4049, 1990.

[25] N. F. Heinig, R. D. Redwing, J. E. Nordman, and D. C. Larbalestier, "Strong to weak coupling transition in low misorientation angle thin film $\mathrm{YBa}_{2} \mathrm{Cu}_{3} \mathrm{O}_{7-\delta}$ bicrystals," Phys. Rev. B, vol. 60, pp. 1409-1417, 1999.

[26] D. T. Verebelyi et al., "Low angle grain boundary transport in $\mathrm{YBa}_{2} \mathrm{Cu}_{3} \mathrm{O}_{7-\delta}$ coated conductors," Appl. Phys. Lett., vol. 76, pp. $1755-1757,2000$.

[27] A. P. Malozemoff, G. N. Riley, S. Fleshler, and Q. Li et al., "Supercurrent conduction mechanisms in BSCCO-2223 tapes," in Proc. Int. Workshop Critical Currents In Superconductors for Practical Applications, Y. H. Kao et al., Eds., 1998, pp. 32-39.

[28] B. Hensel, G. Grasso, and R. Flukiger, "Aspects of the current understanding of the supercurrent transport in $(\mathrm{Bi}, \mathrm{Pb})_{2} \mathrm{Sr}_{2} \mathrm{Ca}_{2} \mathrm{Cu}_{3} \mathrm{O}_{10-x}$ silver-sheathed tapes-The 'railway-switch' model," J. Electron. Mater, vol. 24, pp. 1877-1881, 1995.

[29] H. Wada, L. F. Goodrich, C. Walters, and K. Tachikawa, Eds., IEC 61 788-1 (1998-02), Superconductivity-Part 1: Critical Current Measurement-DC Critical Current of $\mathrm{Cu} / \mathrm{Nb}-\mathrm{Ti}$ Composite Superconductors, IEC 61 788-2 (1999-03), Superconductivity-Part 2: Critical Current Measurement-DC Critical Current of $\mathrm{Nb}_{3} \mathrm{Sn}$ Composite Superconductors.

[30] L. A. Schwartzkopf et al., "The use of the in-field critical current density, $\mathrm{Jc}(0.1 \mathrm{~T})$, as a better descriptor of $(\mathrm{Bi}, \mathrm{Pb})_{2} \mathrm{Sr}_{2} \mathrm{Ca}_{2} \mathrm{Cu}_{3} \mathrm{O}_{x} / \mathrm{Ag}$ tape performance," Appl. Phys. Lett., vol. 75 , pp. 3168-3170, 1999.

[31] G. Stejic et al., "Effect of geometry on the critical currents of thin films," Phys. Rev. B, vol. 49, pp. 1274-1288, 1994.

[32] A. A. Polyanskii et al., "Magneto-optical studies of the uniform critical state in bulk $\mathrm{MgB}_{2}$," Supercond. Sci. Technol., vol. 14, pp. 811-815, 2001.

[33] J. M. Rowell, "The widely variable resistivity of $\mathrm{MgB}_{2}$ samples," Supercond. Sci. Technol., vol. 16, pp. R17-R27, 2003.

[34] M. N. Wilson, C. R. Walters, J. D. Lewin, and P. F. Smith, "Experimental and theoretical studies of filamentary superconducting composites," J. Physics D, Appl. Phys., vol. 3, pp. 1517-1583, 1970.

[35] D. C. Larbalestier et al., "High critical current densities in industrial scale composites made from high homogeneity $\mathrm{Nb} 46.5 \mathrm{Ti}$," IEEE Trans. Mag., vol. 21, pp. 269-272, Mar. 1985.

[36] D. C. Larbalestier, L. Chengren, W. Starch, and P. J. Lee, "Limitation of critical current density by intermetallic formation in fine filament Nb-Ti superconductors," IEEE Trans. Nucl. Sci., vol. NS-32, pp. 3743-3745, 1985.

[37] M. T. Taylor et al., "Co-processed Nb-25\% Zr/Cu composite," Cryogenics, vol. 11, pp. 224-226, 1971.

[38] E. Gregory, T. S. Kreillick, A. K. Ghosh, and W. B. Sampson, "Importance of spacing in the development of high current densities in multifilamentary superconductors," Cryogenics, vol. 27, pp. 178-182, 1987. 
[39] A. W. West, W. H. Warnes, D. L. Moffat, and D. C. Larbalestier, "Compositional inhomogeneities in $\mathrm{Nb}-\mathrm{Ti}$ and its alloys," IEEE Trans. Magn., vol. 19, pp. MAG-749-753, May 1983.

[40] P. J. Lee and D. C. Larbalestier, "Development of nanometer scale structures in composites of $\mathrm{Nb}-\mathrm{Ti}$ and their effect on the superconducting critical current density," Acta Metallurgica, vol. 35, pp. 2523-2536, 1987.

[41] L. Chengren and D. C. Larbalestier, "Development of high critical current densities in niobium 46.5 wt\% titanium," Cryogenics, vol. 27, pp. 171-177, 1987.

[42] G. L. Dorofejev, E. Yu. Klimenko, and S. V. Frolov, "Artificial pinning centers," in Proc. 9th Conf. Magnet Technology, C. Marinucci and P. Weymuth, Eds., 1985, pp. 564-566.

[43] L. D. Cooley and L. R. Motowidlo, "Advances in high field superconducting composites by addition of artificial pinning centers to niobium-titanium," Supercond. Sci. Technol., vol. 12, pp. R135-R151, 1999.

[44] L. R. Motowidlo, M. K. Rudziak, and T. Wong, "The pinning strength and upper critical fields of magnetic and nonmagnetic artificial pinning centers in Nb47wt\% Ti wires," IEEE Trans. Appl. Superconduct., vol. 13, pp. 3351-3354, June 2003.

[45] O. V. Chernyi et al., "The microstructure and critical current density of $\mathrm{Nb}-48 \mathrm{wt} \% \mathrm{Ti}$ superconductor with very high alpha-Ti precipitate volume and very high critical current," Adv. Cryogenic Eng., vol. 48, no. B, pp. 883-890, 2002.

[46] ITER Web page [Online]. Available: http://www.iter.org

[47] N. Mitchell et al., "Strand production and benchmark testing for the ITER model coils," IEEE Trans. Appl. Superconduct., vol. 5, pp. 905-908, June 1995.

[48] R. M. Scanlan, "Conductor development for high energy physics-Plans and status of the U.S. program," IEEE Trans. Appl. Superconduct., vol. 11, pp. 2150-2155, Mar. 2001.

[49] J. A. Parrell, M. B. Field, Y. Zhang, and S. Hong, " $\mathrm{Nb}_{3} \mathrm{Sn}$ conductor development for fusion and particle accelerator applications," Adv. Cryogenic Mater, vol. 50, 2004, to be published.

[50] A. F. Lietzke et al., "Test results for HD1, a $16 \mathrm{~T} \mathrm{Nb}_{3}$ Sn dipole magnet," IEEE Trans. Appl. Superconduct., vol. 14, 2004, to be published.

[51] P. J. Lee et al., "The microstructure and microchemistry of high critical current $\mathrm{Nb}_{3} \mathrm{Sn}$ strands manufactured by the bronze, internal-Sn, and PIT techniques," IEEE Trans. Appl. Superconduct., vol. 13, pp. 3422-3425, June 2003.

[52] J. Lindenhovius et al., "Powder-in-tube (PIT) $\mathrm{Nb}_{3} \mathrm{Sn}$ conductors for high field magnets," IEEE Trans. Appl. Superconduct., vol. 10, pp. 975-978, Mar. 2000

[53] K. Okuno et al., "Test of the NbAl insert and ITER central solenoid model coil," IEEE Trans. Appl. Superconduct., vol. 13, pp. 1437-1440, June 2003.

[54] T. Takeuchi, " $\mathrm{Nb}_{3} \mathrm{Al}$ conductors for high-field applications," Supercond. Sci. Technol., vol. 13, pp. R101-R119, 2000.

[55] N. Banno et al., "Multifilamentary Nb/Al-Ge and Nb/Al-Si precursor fabrication using the intermediately rapid heating and quenching technique," Supercond. Sci. Technol., vol. 17, pp. 320-326, 2004.

[56] F. Buta, M. D. Sumption, and E. W. Collings, "Influence of transformation heat treatment on microstructure and defects in RHQTprocessed $\mathrm{Nb}_{3} \mathrm{Al}$," IEEE Trans. Appl. Superconduct., vol. 13, pp. 3458-3461, June 2003.

[57] T. Hasegawa et al., "High $J_{c} \mathrm{Bi}-2212 / \mathrm{Ag}$ multilayer tape conductor prepared by a coating method," in Advances in Superconductivity XII, T. Yamashita and K. Tanabe, Eds, Tokyo, Japan: Springer-Verlag, 2000, pp. 640-645.

[58] H. W. Weijers et al., "Development and testing of a 3 T Bi-2212 insert magnet for high field NMR," IEEE Trans. Appl. Superconduct., vol. 9, pp. 563-566, June 1999.

[59] T. Kiyoshi et al., "Generation of $23.4 \mathrm{~T}$ using two Bi-2212 insert coils," IEEE Trans. Appl. Superconduct., vol. 10, pp. 472-477, Mar. 2000.

[60] Y. Aoki et al., "A high- $T_{c}$ superconducting Rutherford cable uing Bi-2212 oxide superconducting round wire," in Advances in Superconductivity XII, T. Yamashita and K. Tanabe, Eds, Tokyo, Japan: Springer-Verlag, 2000, pp. 827-829.

[61] R. Gupta et al., "Status of high temperature superconductor magnet R\&D at BNL," IEEE Trans. Appl. Superconduct., vol. 13, pp. 1351-1354, June 2003.

[62] C.-E. Bruzek et al., "High-performance Bi2212/Ag tape produced at Nexans," Supercond. Sci. Technol., 2004, to be published.
[63] S. Patnaik et al., "Local measurement of current density by magneto-optical current reconstruction in normally and overpressure processed Bi-2223 tapes," IEEE Trans. Appl. Superconduct., vol. 13, pp. 2930-2933, June 2003.

[64] Y. B. Huang et al., "Improving the critical current density in Bi-2223 wires via a reduction of the secondary phase content," IEEE Trans. Appl. Superconduct., vol. 13, pp. 3038-3041, June 2003.

[65] Y. Yuan et al., "Overpressure processing of Ag-sheathed $(\mathrm{Bi}, \mathrm{Pb})_{2} \mathrm{Sr}_{2} \mathrm{Ca}_{2} \mathrm{Cu}_{3} \mathrm{O}_{x}$ tapes," Physica $C$, vol. 372-376, pp. 883-886, 2002.

[66] Y. Yuan et al., "Significantly enhanced critical current density in Ag-sheathed $(\mathrm{Bi}, \mathrm{Pb})_{2} \mathrm{Sr}_{2} \mathrm{Ca}_{2} \mathrm{Cu}_{3} \mathrm{O}_{10-x}$ composite conductors prepared by overpressure processing," Appl. Phys. Lett., vol. 84, pp. 2127-2129, 2004.

[67] Y. Iijima et al., "Reel to reel continuous formation of Y-123 coated conductors by IBAD and PLD," IEEE Trans. Appl. Superconduct., vol. 11, pp. 2816-2821, Mar. 2001.

[68] X. D. Wu et al., "Properties of $\mathrm{YBa}_{2} \mathrm{Cu}_{3} \mathrm{O}_{7-\delta}$ thick-films on flexible buffered metallic substrates," Appl. Phys. Lett., vol. 67, pp. 2397-2399, 1995.

[69] A. Usoskin et al., "Large area YBCO-coated stainless steel tapes with high critical currents," IEEE Trans. Appl. Superconduct., vol. 13, pp. 2452-2457, June 2003.

[70] S. R. Foltyn et al., "Strongly coupled critical current density values achieved in $\mathrm{YBa}_{2} \mathrm{Cu}_{3} \mathrm{O}_{7-\delta}$ coated conductors with near-single-crystal texture," Applied Physics Letters, vol. 82, pp. 4519-4521, 2003.

[71] T. Izumi, Y. Yamada, and Y. Shiohara, "All Japan efforts on R\&D of HTS coated conductors for future applications," Physica C, vol. 392-396, pp. 9-16, 2003.

[72] A. Goyal et al., "High critical current density superconducting tapes by epitaxial deposition of $\mathrm{YBa}_{2} \mathrm{Cu}_{3} \mathrm{O}_{x}$ thick films on biaxially textured metals," Appl. Phys. Lett., vol. 69, pp. 1795-1797, 1996.

[73] A. Goyal et al., "Recent progress in the fabrication of high- $J_{c}$ tapes by epitaxial deposition of YBCO on RABiTS," Physica $C$, vol. 357-360, pp. 903-913, 2001.

[74] K. Ohmatsu et al., "Development of in-plane aligned YBCO tapes fabricated by inclined substrate deposition," Physica C, vol. 357-360, pp. 946-951, 2001.

[75] A. P. Malozemoff et al., "Low-cost YBCO coated conductor technology," in IOP Conf. Series, vol. 167, London, 2000, pp. 307-312.

[76] D. Verebelyi et al., "Practical neutral axis conductor geometries for coated conductor composite wire," Superconductor Science and Technology, vol. 16, pp. 1158-1161, 2003. ibid, "Uniform performance of continuously processed MOD-YBCO coated conductors using a textured Ni-W substrate,"Supercond. Sci. and Technol., pp. L19-L23, vol. 16, 2003.

[77] C. P. Wang et al., "Deposition of in-plane textured $\mathrm{MgO}$ on amorphous $\mathrm{Si}_{3} \mathrm{~N}_{4}$ substrates by ion-beam-assisted deposition and comparison with ion-beam-assisted deposition yttria-stabilized-zirconia," Appl. Phys. Lett., vol. 71, pp. 2955-2957, 1997.

[78] R. Metzger, "Superconducting tapes using ISD buffer layers produced by evaporation of $\mathrm{MgO}$ or reactive evaporation of magnesium," IEEE Trans. on Applied Superconductivity, vol. 11, pp. 2826-2829, Mar. 2001.

[79] B. Ma et al., "High critical current density of YBCO coated conductors fabricated by inclined substrate deposition," Physica C, vol. 403, pp. 183-190, 2004.

[80] R. P. Reade, P. Berdahl, R. E. Russo, and S. M. Garrison, "Laser deposition of biaxially textured yttria-stabilized zirconia buffer layers on polycrystalline metallic alloys for high critical current Y-Ba-Cu-O thin films," Appl. Phys. Lett., vol. 61, pp. 2231-2233, 1992.

[81] V. Selvamanickam, H. G. Lee, Y. Li, X. Xiong, Y. Qiao, J. Reeves, Y. Xie, A. Knoll, and K. Lenseth, "Fabrication of 100 A class, $1 \mathrm{~m}$ long coated conductor tapes by metal organic chemical vapor deposition and pulsed laser deposition," Physica C, pp. 392-396, 859-862, 2003.

[82] D. M. Feldmann et al., "Through-thickness superconducting and normal-state transport properties revealed by thinning of thick film ex situ $\mathrm{YBa}_{2} \mathrm{Cu}_{3} \mathrm{O}_{7-\delta}$ coated conductors," Appl. Phys. Lett., vol. 83, pp. 3951-3953, 2003.

[83] A. P. Malozemoff. (2004) Second Generation HTS Wire: An Assessment. [Online]. Available: http://www.amsuper.com/html/products/library/2g_white_paper_-_march_2002.pdf

[84] J. Nagamatsu et al., "Superconductivity at $39 \mathrm{~K}$ in magnesium diboride," Nature, vol. 410, pp. 63-64, 2001. 
[85] H. J. Choi et al., "The origin of anomalous superconducting properties of $\mathrm{MgB}_{2}$, "Nature, vol. 418, pp. 758-760, 2002.

[86] A. Gurevich, "Enhancement of $H_{c 2}$ by nonmagnetic impurities in dirty two-gap superconductors," Phys. Rev. B, vol. 67, pp. $185415-13,2003$.

[87] A. Gurevich et al., "Very high upper critical fields in $\mathrm{MgB}_{2}$ produced by selective tuning of impurity scattering," Supercond. Sci. Technol., vol. 17, pp. 278-286, 2004.

[88] D. C. Larbalestier et al., "Strongly linked current flow in polycrystalline forms of the new superconductor $\mathrm{MgB}_{2}$, , Nature, vol. 410, pp. 186-189, 2001.

[89] Physica C, vol. 385, no. 1-2, pp. 1-385, Mar. 2003. A broad review of recent work on $\mathrm{MgB}_{2}$.

[90] V. Braccini et al. (2004) High-field superconductivity in alloyed $\mathrm{MgB}_{2}$ thin films. Condens. Matter [Online]. Available: http://arxiv.org/pdf/cond-mat/0402002

[91] W. Goldacker et al., "Considerations on critical currents and stability of $\mathrm{MgB}_{2}$ wires made by different preparation routes," Physica C, vol. 401, pp. 80-86, 2004.

[92] R. F. Klie et al., "Observation of coherent oxide precipitates in polycrystalline $\mathrm{MgB}_{2}$," Appl. Phys. Lett., vol. 80, p. 3970, 2002.

[93] X. Song, V. Braccini, and D. Larbalestier, "Inter- and intra-granular nanostructure and possible spinodal decomposition in low resistivity bulk $\mathrm{MgB}_{2}$ with varying critical fields," J. Mater. Res., 2004, to be published.

[94] S. Y. Xu et al., "High critical current density and vortex pinning of epitaxial $\mathrm{MgB}_{2}$ thin films," Phys. Rev. B., vol. 68, pp. 224 501-224 508, 2003.

[95] V. Braccini and D. C. Larbalestier, unpublished work.

[96] L. D. Cooley and R. M. Scanlan, private communication.

[97] Y. Ando et al., "Resistive upper critical fields and irreversibility lines of optimally doped high- $T_{c}$ cuprates," Phys. Rev. B, vol. 60, pp. 12 475-12479, 1999.

[98] R. H. T. Wilke et al.. (2003) Systematic effects of carbon doping on the superconducting properties of $\operatorname{Mg}\left(\mathrm{B}_{1-x} \mathrm{C}_{x}\right)_{2}$. Condens. Matter Archive [Online]. Available: http://arxiv.org/abs/cond-mat/0312235

[99] E. Ohmichi, T. Masui, S. Lee, S. Tajima, and T. Osada. (2003) Enhancement of the irreversibility field by carbon substitution in single crystal $\mathrm{MgB}_{2}$. Condens. Matter Archive [Online]. Available: http://arxiv.org/abs/cond-mat/0312348

[100] S. X. Dou et al., "Enhancement of the critical current density and flux pinning of $\mathrm{MgB}_{2}$ superconductor by nanoparticle $\mathrm{SiC}$ doping," Appl. Phys. Lett., vol. 81, pp. 3419-3421, 2002.

[101] S. Soltanian et al., "Transport critical current of solenoidal $\mathrm{MgB}_{2} / \mathrm{Cu}$ coils fabricated using a wind-reaction in situ technique," Supercond. Sci. Technol., vol. 16, pp. L4-L6, 2003.

[102] G. Grasso et al., "Fabrication and properties of monofilamentary $\mathrm{MgB}_{2}$ superconducting tapes," Supercond. Sci. Technol., vol. 16, pp. 271-275, 2003.

[103] A. Matsumoto, H. Kumakura, H. Kitaguchi, and H. Hatakeyama, "Effect of impurity additions on the microstructures and superconducting properties of in situ-processed $\mathrm{MgB}_{2}$ tapes," Supercond. Sci. Technol., vol. 17, pp. S319-S323, 2004.

[104] S. Kalsi et al., "Superconducting dynamic synchronous condenser for improved grid voltage support," presented at the IEEE Transmission and Distribution Conf., Dallas, TX, 2003.

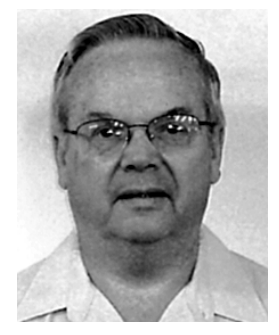

Ronald M. Scanlan received the Ph.D. degree from Cornell University, Ithaca, NY, in 1970.

Earlier in his career, he was responsible for development of $\mathrm{Nb}_{3} \mathrm{Sn}$ conductor for the HFTF fusion magnet at the Lawrence Livermore National Laboratory. He was the Group Leader for Superconducting Wire and Cable Development at the Lawrence Berkeley National Laboratory (LBNL), Berkeley, CA. At LBNL (1994-1999), he also served as the Magnet Program Head during the design, construction, and testing of the world's first 13.5- $\mathrm{T} \mathrm{Nb}_{3}$ Sn dipole magnet. Most recently, he managed the Conductor Development Program for the Division of High Energy Physics, U.S. Department of Energy, Washington, DC. The goal of this program is the industrial development of a cost-effective, high-field superconductor for accelerator magnet applications. In the first 18 months of this program, one of the participating industrial firms was able to increase the critical current density in $\mathrm{Nb}_{3} \mathrm{Sn}$ by over $50 \%$, to $3000 \mathrm{~A} / \mathrm{mm}^{2}$ at $4.2 \mathrm{~K}$ and $12 \mathrm{~T}$. He is currently retired. He is the author or coauthor of over 100 publications in the field of superconducting materials.

Dr. Scanlan shared the IEEE Particle Accelerator Conference Award for 1991 with Dr. D. C. Larbalestier "for the development of NbTi superconducting material for high current density application in high field superconducting magnets."

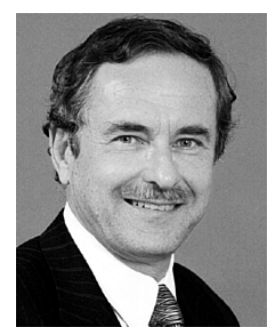

Alexis P. Malozemoff (Senior Member, IEEE) received the B.A. degree from Harvard University, Cambridge, MA, in 1966 and the Ph.D. degree from Stanford University, Stanford, CA, in 1970.

He was with IBM Research, Yorktown Heights, NY. He is currently Executive Vice President and Chief Technical Officer of American Superconductor Corporation, Westborough, MA. He is responsible for research and development strategy, advanced conductor development, and the intellectual property portfolio. He has more than 20 years of experience in superconducting materials and systems, as well as in magnetic materials and devices. He has numerous publications and patents in magnetism and superconductivity.

Dr. Malozemoff is a Fellow of the American Physical Society.

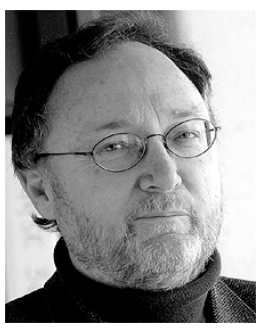

David C. Larbalestier received the Ph.D. degree from Imperial College, London, U.K., in 1970.

He worked in the Superconducting Magnet Research Group of the Rutherford Laboratory in $1972-1976$ on the first multifilamentary $\mathrm{Nb}_{3} \mathrm{Sn}$ conductors and magnets. He joined the University of Wisconsin, Madison, in 1976, where he is currently Professor in the Departments of Materials Science and Engineering and of Physics, where he holds both the L. V. Shubnikov Chair and the Grainger Chair of Superconductivity and is also the Director of the Applied Superconductivity Center. He has been active in superconductivity since he was working toward his Ph.D. degree. His group has had a large influence on the understanding and application of both low and high temperature superconductors.

Dr. Larbalestier is a Fellow of the American Physical Society and the Institute of Physics and a Member of the National Academy of Engineering. His Ph.D. work gained the Matthey Prize of Imperial College. He shared a 1978 IR-100 award with an Oxford Instrument team for their work on the first $\mathrm{Nb}_{3} \mathrm{Sn}$ NMR magnet. His work has been recognized by the 1991 IEEE Particle Accelerator Conference Award (jointly with R. M. Scanlan) and the 2000 IEEE Award for continuing and significant contributions in the field of applied superconductivity, and by the Council for Chemical Research (2000) for work by him and his collaborators on $(\mathrm{Bi}, \mathrm{Pb})_{2} \mathrm{Sr}_{2} \mathrm{Ca}_{2} \mathrm{Cu}_{3} \mathrm{O}_{x}$. 